\title{
10 „Der Kampf um die Arbeitsfreude“: Arbeitspsychologisches Glückswissen zur Zeit der Weimarer Republik
}

\section{1 „Wille zur Arbeitsfreude“: Autosuggestive „Glücksgesinnung“ im Ratgeber von Richard Baerwald}

\begin{abstract}
„Es fehlt uns jene Grundvoraussetzung aller Lebensweisheit, die ich als ,Glücksgesinnung“ bezeichnen möchte. Unter Glücksgesinnung bei der Arbeit verstehe ich die Absicht, unbedingt aus jeder noch so geringen Tätigkeit Freude und Genuß zu ernten [...]. Dieser Wille zur Arbeitsfreude ist viel wichtiger als die später zu besprechenden technischen Hilfsmittel, die uns die Arbeit versüßen. [...] Die suggestive Macht dieses Verfahrens in Fällen, in denen uns vor einer Arbeit graut, kann nicht hoch genug veranschlagt werden.“1
\end{abstract}

In seinem Ratgeber „Lebensmut, Erfolg, Arbeitsfreude. Der Weg zu Glück und Zuversicht“ von 1921 appelliert der Psychologe Dr. Richard Baerwald (1867-1929) auf der Grundlage der angewandten Arbeitspsychologie an den „Wille[n] zur Arbeitsfreude“ und die „suggestive Macht“ einer „Glücksgesinnung“ bei der Arbeit. ${ }^{2}$ Darunter versteht der Autor eine autosuggestive Praktik, die es mittels einer leistungsorientierten Selbsterziehung ermögliche, sogar in den eintönigsten Arbeiten „Glück“ zu empfinden. ${ }^{3}$

Wie stellte sich der Ratgeberautor Richard Baerwald seine „Glücksgesinnung“, eine Praktik positiver Autosuggestion, im Alltag vor? Die psychologische Überlistung des inneren Arbeitswiderstands gelinge, sobald nicht länger das unerreichbare Fernziel, sondern die Aufteilung der Tätigkeit in kurzzeitige Etappenziele in den Blick genommen würde. Diese gerichtete Wahrnehmung und die

1 Baerwald 1933, $232 \mathrm{f}$.

2 Vgl. ebd., 232. Baerwald zitiert in seinem interdisziplinär angelegten Ratgeber neben Komponisten (Beethoven, Johannes Brahms, Joseph Haydn, Felix Mendelssohn-Bartholdy, Richard Wagner), Dichtern, Dramatikern und Schriftstellern (Charles Dickens, Theodor Fontane, Johann W. v. Goethe, Maxim Gorki, Grillparzer, Keller, Thomas Mann, Schiller, Hermann Sudermann, August Strindberg, Lew Nikolajewitsch Tolstoi, Franz Wedekind, Émil Zola) mehrheitlich Wissenschaftler, u. a. Wilhelm von Bode (1845 - 1929), Eugen Düring (1833-1921), Horatio W. Dresser (1866-1954), Ernst Frh. v. Feuchtersleben (1806-1849), Francis Galton (1822-1911), Eduard von Hardmann (1842-1906), Karl Hilty (1833-1909), William James (1842-1910), Ellen Key (18491826), Sir John Lubbock (1834-1913), [Johannes Jaroslaw] Marcinowski (1868-1935), Prentice Mulford (1834-1891), Franz Müller-Lyer (1857-1916), Friedrich Paulsen (1846-1908), Jules Payot (1859-1939), Gustav v. Schmoller (1838-1917) Oswald Spengler (1880-1936). Vgl. Namens- und Sachregister Baerwald 1933, 323-331.

3 Vgl. Baerwald 1933, 233.

Ә OpenAccess. (c) 2021 Isabelle Haffter, publiziert von De Gruyter. (cc))BY-NC-ND Dieses Werk ist lizenziert unter einer Creative Commons Namensnennung - Nicht kommerziell - Keine Bearbeitung 4.0 International Lizenz. https://doi.org/10.1515/9783110661439-010 
damit verbundene aufmerksame Hinwendung auf die eigene Fertigkeit im Moment des Arbeitsschrittes könne eine genussvolle „Freude“ am „Selbstzweck“ auslösen. ${ }^{4}$ Baerwalds Leitsatz lautet daher „Die Freude am Schaffen als Selbstzweck“ ${ }^{“ 5}$, die aus „Fronarbeit“ „Froharbeit“6 bzw. „Arbeitsglück ${ }^{\text {“7 }}$ generiere.

Wen wollte der Psychologe mit diesem Leitsatz erreichen? Oder anders gefragt, an welche Leserschaft richtete sich sein Ratgeber? Die arbeitspsychologische Praktik der Autosuggestion sei besonders für monotone Fabrikarbeiten relevant, womit er auf sein Zielpublikum, die Arbeiterschaft, hinweist. Der „Wert“ ihrer Arbeit liege im „Arbeiten selber, in der Tätigkeit, im Streben und Ringen[...]“. 8

Auf welches Wissen griff Baerwald beim Entwurf seiner Gesinnung eines „Streben[s] und Ringen[s]“ um „Glück“ zurück? Wer den „Willen“ zur „Arbeitsfreude“ besäße, verspricht der Autor euphorisch, der könne auch „Liebe zum Schicksal“, zum monotonen Arbeitsschicksal, empfinden. ${ }^{9}$ Frei nach Nietzsches Aphorismus über „Amor fati“10, der höchsten, menschlichen Lebensbejahung, setzt Baerwald die „Erziehung der Schicksalsliebe“11 mit der „Erziehung zur Glücksgesinnung“12 gleich: „,Amor fati“ läßt uns in jeder Arbeit, die wir vor uns haben[,] mit Fleiß die Lichtpunkte heraussuchen und mit der Aufmerksamkeit betonen. "13 Nicht Eudämonie, eine errungene Glücksseligkeit, sei das höchste Lebensziel des Menschen, sondern seine Liebe zum schicksalshaften Arbeitsglück. ${ }^{14}$

4 Vgl. ebd., 217, 232.

5 Ebd., 217-281.

6 Zur „Froharbeit und Frohnarbeit“" vgl. ebd., 278-281.

7 Vgl. u. a. ebd., 271.

8 Vgl. ebd., 218.

9 Vgl. ebd., $232 \mathrm{f}$.

10 Baerwald zitiert mit orthografischen und inhaltlichen Textänderungen den bekannten 276. Aphorismus „Zum neuen Jahre“ von 1882 über die „amor fati“ wie folgt: „,Textauslassung] Ich will immer mehr lernen, das Notwendige [„Nothwendige“] an den Dingen als das Schöne zu sehen [\} „Zu“] : [,,““] so werde ich einer [,Einer“] von denen [„Denen“] sein, welche die Dinge schön machen. Amor fati: Das [,das“] sei von nun an meine Liebe [,!"“] ... [Textauslassung]. Ich will nicht anklagen; [ „;““, Textauslassung] Wegsehen [,We g s e h en “] sei meine einzige Verneinung! Und Alles in Allem und Großen: ich will irgendwann einmal nur noch ein Jasagender sein!“ Vgl. Baerwald 1933, 304. [Textabgleich vgl. Nietzsche 1988, 521 („§ 276. [„Zum neuen Jahre, Genua im Januar 1882"]. Die fröhliche Wissenschaft).

11 Baerwald 1933, 311.

12 Ebd., 232.

13 Vgl. Baerwald 1933, 232.

14 Vgl. ebd., 218f., 271. 
Es käme aber nicht nur auf das Individuum und dessen alleinigen „Wille[n] zur Arbeitsfreude“ an, sondern auch auf das Arbeitskollektiv. Ein entscheidendes Gefühlselement der „Glücksgesinnung“ sei ein soziales Solidaritätsgefühl, das sich unter Arbeitskolleg*innen einstellen könne. ${ }^{15}$ Darunter versteht der Autor „die schöne Wirkung, daß das gemeinsame Interesse und das Hinzugehören zu einem größeren Verband Berufsstolz und Wichtignehmen der Arbeit unterstützt." 16

Nach der Lektüre von Baerwalds Glücksratgeber drängt sich die Frage auf, welche praktisch umsetzbaren Leitsätze aus den mitunter assoziativen und autobiografisch gefärbten Gedankenspiralen des Ratgeberautors der Leserschaft empfohlen wurden. Erwin Heine hält in einem Kommentar an die Leser^innen der zweiten Auflage von 1933 fest, dass Baerwalds „Erziehung zur Schicksalsliebe“ eine „glückschaffende Phantasie“17 eines „sozialen Zukunftsstaat[s]“18 sei. Die Frage nach der Leserrezeption drängt sich an dieser Stelle auf.

Wilhelm Thimme (1879-1966), der evangelisch-lutherische Theologe und habilitierte Lehrbeauftragte für Religionspsychologie an der Westfälischen Wilhelms-Universität in Münster, äußerte Zweifel an der Wirkungsmacht von Baerwalds Glückswissenspraktik. ${ }^{19}$ Die Idee einer „Kultur der Arbeitslust“ in Form eines autosuggestiven „Studium[s]“ fand er durchaus interessant. ${ }^{20}$ Auch Baerwalds eigens erprobte „Lebensmethode“ hielt der Theologe für authentisch.

15 Vgl. Baerwald 1933, 236.

16 Vgl. ebd., 236. Hier bestehen Anknüpfungspunkte zur NS-Arbeitsideologie, worauf insb. in Kap. 10.4 und 12 näher eingegangen wird.

17 Vgl. Baerwald 1933, 316. Erwin Heine schreibt im Vorwort zur Zweitausgabe (1933), dass er zum besseren Verständnis Baerwalds Ratschläge, die angeblich „schwer verständliche philosophische Fachausdrücke und andere Fremdwörter“ enthielten, in „gemeinverständliches Deutsch übertragen“ hätte. Zur „leichteren Übersicht“ hätte er zudem kurze Randnotizen mit knappen Inhaltsangaben hinzugefügt, „,gesonderte Betitelung“ gewählt, Einzelpassagen in „Fettdruck“ gesetzt sowie ein Schlagwort-, Namen- und Sachverzeichnis zur Erklärung hinzugefügt. „In seinem neuen volkstümlichen Gewand“, schreibt Heine im Herbst 1932, hoffe er, dass Baerwalds Schrift „seine hohe und notwendige Sendung erfüllen wird: ein Wegweiser zu sein zu Glück und Zuversicht“. Vgl. ebd. 1933, 7. Aufschlussreich an diesem Vorwort ist die Annahme Heines, dass ein Ratgeber in einer zu akademisch gehaltenen Sprache nicht breit rezipiert werden würde. Außerdem gibt Heine Auskunft über das Leseverhalten des Zielpublikums, indem man implizit erfährt, dass sich die Leserschaft in kurzer Zeit anhand schlagwortartiger Randbemerkungen einen Überblick über die Ratschläge verschaffen und sich in keinem langwierigen Lektürestudium die Lehrsätze selbst erarbeiten möchte.

18 Vgl. Baerwald 1933, 316.

19 Zur Biografie vgl. Foerster 1974.

20 Vgl. Thimme 1922. 
Dennoch war Thimme skeptisch „[...] ob er sich nicht doch einer Illusion hingibt?“21 Was waren seine Einwände? „Glaube“ sei, laut Thimme, nicht mit „technisch-autosuggestiver [...] Klugheit und Geschicklichkeit“ zu erwirken. Dem christlichen „Glauben“ zumindest liege ein idealistischer Harmoniegedanke zugrunde. Dieser stände in einem unauflösbaren Widerspruch zu Baerwalds arbeitspsychologischer Praktik einer leistungsorientierten Autosuggestion. Darin sah Thimme das Grundproblem der „Gemütskurbewegung“, zu deren Vertretern er Baerwald zählte. ${ }^{22}$

Wie wurde Baerwalds Glückswissens-Lehre von seinen Fachkolleg^innen rezipiert? Als Mitglied der „Psychologischen Gesellschaft zu Berlin“ konnte Baerwald vor seinen Berufskolleg^innen am 20. Oktober 1921 einen Vortrag „über Arbeitsfreude, ihre psychologischen Bedingungen und künstliche Weckung“23 halten. ${ }^{24}$ Dabei handelte es sich um Auszüge aus seiner im selben Jahr noch erschienenen Schrift „Arbeitsfreude und andere Beiträge zur psychologischen Lebenskunst“ ${ }^{25}$ Dem Sitzungsbericht von Rechtsanwalt Westmann zufolge war die zentrale Botschaft an die Wissenschaftler*innen dieselbe, wie an das Ratgeberpublikum: Eine „Arbeitsfreude“ könne durch „planvolle Autosuggestion“ erreicht werden, namentlich mittels „technisch-psychologische[r]“ „Kniffe‘, wie sie Westmann trickreich umschreibt, „durch die man sich seine Arbeit geniessbar machen“26 könne. Auch lästige Arbeiten könnten auf diese Weise „Vergnügen“ bereiten, wenn man, statt sich ihnen zu widersetzen, in ihnen aufgehe:

„[...] jeder Einzelbewegung ihr zukommendes Aufmerksamkeitsquantum gönnt, so dass sie exakt und präzis abläuft und das Gefühl des Könnens und Gutmachens in uns auslöst. Alles kann Vergnügen werden, wenn man es ebenso zum Selbstzweck werden lässt wie es das

21 Vgl. ebd.

22 Vgl. ebd.

23 Weitere Vortragstitel lauteten „Musikalische Erinnerungsverklärung“ (19.11.1914) vgl. Sitzung vom 19. November 1914. Sitzungsberichte. Psychologische Gesellschaft zu Berlin. In: Westmann 1920, 93, mit dem Publikationshinweis auf die „Zeitschrift für Aesthetik und allgemeine Kunstwissenschaft“, in der Baerwald mehrfach publizierte u.a. „,Zur Psychologie des Komischen“, vgl. Baerwald 1914 und Baerwald 1907; „Die Unbeliebtheit des Tüchtigen (Psychologische Glossen zur Isolierung Deutschlands)“ (20.05.1915), vgl. Sitzung vom 20. Mai 1915. In: Westmann 1920, 98; „Lebenskunst und Gefühlsbeherrschung in ernster Zeit“ (03.05.1917) zur Methode der „psychischen Distanz“, vgl. Sitzung vom 3. Mai 1917. In: Westmann 1920, 113; „Die Schwächen des deutschen Nationalgefühls als völkerpsychologisches Problem“ (25.11.1920) mit dem Publikationshinweis, vgl. Baerwald 1921. Vgl. Sitzung vom 25. November 1920. In: Westmann 1922, 239.

24 Vgl. Sitzung vom 20. Oktober 1921. In: Westmann 1922, 243.

25 Vgl. Baerwald 1921.

26 Vgl. Sitzung vom 20. Oktober 1921. In: Westmann 1922, 244. 
Vergnügen zu sein pflegt. - Frohe Arbeit wirkt kraftsparend, Interesse und Freude bilden daher, trotz [Emil] Kraepelins nur teilweise begründetem Einspruch, den besten Schutz gegen Uebermüdung und Ueberanstrengung. “27

Was lässt sich zusammenfassend zur wissenshistorischen Erkenntnis über das arbeitspsychologische Glückswissen bei Baerwald festhalten? Baerwalds Ratgeber zu „Glück und Zuversicht“ ist eine Anleitung zur lebensbejahenden und doch schicksalsergebenen, „planmäßige[n] Autosuggestion“28. Im Rückgriff auf tradiertes Glückswissen über Willenskraft und Lebensführung aus der pädagogischen und moralphilosophischen Reformbewegungszeit beschwor Baerwald mittels „tröstend[en], wegglättend[en], einschmeichelnd[en] Reden“29 einerseits die individuelle „Selbstbeeinflussung“30 ${ }^{\text {, }}$ andererseits die gemeinschaftsstiftende Wirkungsmacht einer kollektiven Glücksgesinnung in der alltäglichen Arbeitswelt. Seine Anleitung zur arbeitspsychologischen Praktik der Autosuggestion zum Erlangen von „Lebensmut, Erfolg und Arbeitsfreude“ werden in den Worten Thimmes als „Kultur der Arbeitslust“ beschrieben und in der vorliegenden Untersuchung als Teil einer Politik der ,Glückskulturen‘ begriffen.

Diese erste Analyse eines Ratgebers als wissenshistorische Quelle diente zur thematischen Einführung. Im Folgenden sollen die Analyseerkenntnisse anhand unterschiedlicher Quellenbeispiele aus dem Bereich der Arbeitswissenschaft vergleichend analysiert und systematisch kontextualisiert werden. Dabei stellen sich eingangs zwei Fragen, die im oben zitierten Sitzungsbericht ebenfalls anklingen: 1. Welches Dilemma sprach der erfahrene Psychiater Emil Kraepelin (1865 - 1926), der bereits 1896 eine arbeitspsychologische Schrift „Zur Hygiene der Arbeit“31 publiziert hatte, in seinem „Einspruch“ an, wenn er „teilweise begrün$\operatorname{det}[. .$. ]“ einwarf, dass selbst „Interesse und Freude“ keinen „Schutz“ vor „[ய̈] bermüdung“ und „[Ü]beranstrengung“ garantieren könnten? ${ }^{32} 2$. Handelte es sich bei Baerwalds Glücksratgeber zur Problematik der „Arbeitsfreude“ um eine Ausnahmeerscheinung oder um ein zeittypisches Phänomen?

27 Ebd.

28 Baerwald 1933, 233.

29 Ebd., 241.

30 Ebd., 241.

31 Kraepelin 1896. Zu Kraeplins Werk vgl. Kraepelin 2000, zu seiner Person vgl. u. a. Hoff 1994.

32 Vgl. Sitzung vom 20. Oktober 1921. In: Westmann 1922, 244. 


\subsection{Rationalisierte „Arbeitsfreude“: Das verhängnisvolle Versprechen der Psychotechnik}

Zur Zeit der Niederschrift seines Ratgebers um 1921 war Baerwald Dozent an der Humboldt-Akademie in Berlin. ${ }^{33}$ Der Umstand, dass ein Psychologe auf der Grundlage wissenschaftlicher Erkenntnisse einen Glücksratgeber für das „arme deutsche Volk“ mit dem „praktischen Zweck des Tröstens und Heilens“ verfasste, war für die Nachkriegszeit nicht ungewöhnlich. ${ }^{34}$ Das Ersehnen eines „Arbeitsglücks“35, beziehungsweise das Bedauern über dessen Abwesenheit, war ein populäres Thema in der massenmedialen Ratgeberliteratur der Weimarer Republik. ${ }^{36}$ Weshalb? Baerwald nennt folgenden möglichen Grund: „[...] nie war eine Epoche der Arbeitsfreude so bitter feindlich wie unser Zeitalter des Kapitalismus, der Organisation, der zentralisierten Produktion.“37

Wie lässt sich diese Begründung wissenshistorisch erklären? Werden heutzutage unter den Schlagworten „Stress“ und „Burnout“ Überarbeitungssymptome als Probleme der westlichen Arbeitsgesellschaft diskutiert, wurden in der Weimarer Republik mit den Begriffen „Ermüdung“38 und „[Ü]beranstrengung“ physische und psychische Belastungen in der Arbeiterschaft problematisiert. ${ }^{39}$ Als

33 Biografischer Hinweis zum Autor vgl. Baerwald 1933, 440.

34 Vgl. ebd., $5 \mathrm{f}$.

35 „Das Arbeitsglück ist dauerhafter als die Freude am Leben“ lautete eine Randbemerkung von Erwin Heine in der zweiten Auflage. Vgl. Baerwald 1933, 271.

36 Eine Auswahl aus Deutschland und der Schweiz (alphabetische Reihenfolge): Förster, Fr[iedrich] W[ilhelm]. 1930. Lebensführung, Erlenbach-Zürich und Leipzig: Rotapfel; Gerling, Reinhold. 1921. Tatmensch. Eine Lebensberatung zu kraftvoller erfolgreicher Lebensgestaltung, 4. Aufl., Prien: Anthropos-Verlag; Grevenberg, E. 1932. Der goldene Faden. Eine Wegleitung durch die Wirrnis des Lebens für Glücks- und Wahrheitssucher. Neugeist-Bücher 45, Pfullingen (Württemberg): Johannes Baum-Verlag; Grossmann, G[ustav]. 1927. Sich selbst rationalisieren. Wesen und Praxis der Vorbereitung persönlicher u. beruflicher Erfolge, 9. Aufl. Stuttgart und Wien: Verlag für Wirtschaft und Verkehr Forkel \& Co; Jung, Franz. 1921. Die Technik des Glücks. Psychologische Anleitungen in vier Übungsfolgen, Berlin: Der Malik-Verlag. Jung, Franz. 1923. Mehr Tempo! Mehr Glück! Mehr Macht! Die Technik des Glücks, II. Teil. Ein Taschenbuch für Jedermann, Berlin: Der Malik-Verlag; Klotz, Karl 1928. Die neue Rasse. Ein Wegweiser zu Höherentwicklung, Glück und Freude, für den Einzelnen, die Familie und die Gesamtheit. LebensbornBücher 22, Bedeutend erweiterte und vollständig umgearbeitete Auflage, Düsseldorf: LebensbornVerlag; Wardall, Max 1928. Gedanken-Radio. Der Mensch als Gedanken-Sender und -Empfänger. Ein praktischer Weg zu Glück, Harmonie und Meisterung des Lebens, Pfullingen (Württemberg): Johannes Baum.

37 Vgl. Baerwald 1933, 220.

38 Vgl. u. a. Ermüdung. In: Münsterberg 1919, 127-131.

39 S. Kaeplins Einwand, vgl. Sitzung vom 20. Oktober 1921. In: Westmann 1922, 244. 
Grund wurde oft die Rationalisierung von Arbeitsprozessen zur Produktionssteigerung genannt. ${ }^{40}$ Was verstand die Arbeitswissenschaft unter „Rationalisierung“ und welcher Zusammenhang bestand in Bezug zur Kultur der „Arbeitsfreude“? Gemäß dem Arbeitspsychologen Fritz Giese (1890-1935), dem Herausgeber des Handwörterbuchs der Arbeitswissenschaft (1927-1930), gab es in der Weimarer Republik keine einheitliche Begriffsdefinition für das Rationalisierungsphänomen. Zum Verständnis des Konzepts war „die vernunftgemäße (durch wissenschaftliche Einsicht praktisch geregelte) intensive Bestgestaltung“ von zentraler Bedeutung. ${ }^{41}$ Darunter verstand Giese den vernunftgemäßen Charakter der Rationalisierung ${ }^{42}$, die sich nach seiner Auffassung von einer „Gefühlspolitik“ als „bloßer Intuition“ unterscheide. ${ }^{43}$ Rationalisierung sei „keine ,Kunst“ und nichts Trickähnliches, sondern eine Lehre, ein wissenschaftlich zu ergründendes und so zur Anwendung (= praktische Gestaltung) strebendes Phänomen““.44 Das Ziel der Rationalisierung sei, im Unterschied zum Taylorismus und Fordismus, „ein Optimum kein Maximum“ der Arbeitsprozesse zu erlangen. Aus diesem Grund sei das Konzept für die aktuelle Arbeitswissenschaft von größter Bedeutung. ${ }^{45}$

Der Arbeitswissenschaftler äußerte Kritik an der praktischen Umsetzung dieser ,vernunftorientierten“ Wissenschaft, die ohne „Gefühlspolitik“ funktioniere und folglich die Gefühlswelt der Arbeiterschaft nicht miteinbeziehe. ${ }^{46}$ Rationalisierung sei bereits heute ein „historisch-praktischer Begriff von Übergangsbedeutung“, ein Zeitphänomen also, wie es (nach Gieses Auffassung) auch der „Kapitalismus“47 darstelle. ${ }^{48}$ Die Anklänge an eine sozialpolitische und humanistische Reformbestrebung im Sinne der sozialdemokratischen Gewerkschaftsverbände, die in seiner auffallend gefühls- und psychopolitisch argumentierenden Kritik aufblitzen, sollte Giese im Zuge seines ideologischen Gesinnungswandels nach 1933 revidieren, wie in den Kapiteln 11 und 12 aufgezeigt wird.

40 Zum Begriff vgl. Giese 1930.

41 Vgl. ebd., 3622.

42 ratio: lat. „Vernunft, schlussfolgender, logischer Verstand“. Zum Begriff, vgl. Art. ratio 2019.

43 Vgl. Giese 1930, 3622.

44 Vgl. ebd., 3622.

45 Vgl. ebd., 3622.

46 Vgl. ebd., 3631. Dieses Argument wird in der Gefühlspolitik der NS-Arbeitsideologie mit Bezug auf die NS-Arbeitswissenschaft relevant, s. dieses Kapitel w.u. und Kap. 7, 11, 12.

47 Hier widersprach Giese den angeblichen Auffassungen Hitlers, der den Kapitalismus ,sinnvoll“ im Interesse des NS-Regimes nutzen wollte. Zu Hitlers Auffassung zum „Kapitalismus“ in einem Gespräch mit August Winnig vgl. Winnig 1946, 39.

48 Vgl. Giese 1930, 3631. 
Gieses Kritik der „Schädigungswirkungen“ zielte auf die Umsetzung der Rationalisierungstheorie in der Arbeitspraxis von medientechnologischen Einrichtungen bei der Deutschen Post (Telefonistinnen) oder maschinellen Großbetrieben wie Siemens, Zeiss oder Krupp. ${ }^{49}$ Schon Baerwald hatte die arbeitswissenschaftliche Rationalisierungspraktik kritisiert und diese zum Anlass genommen, seinen Glücksratgeber zu verfassen: „Neben den zu schwierigen Arbeiten, die über unsere Kraft gehen, bilden die größte Folter jene ganz monotonen und maschinellen, wie sie die moderne Arbeitsteilung namentlich über den Fabrikarbeiter verhängt, Tätigkeiten, die unsere höheren geistigen Kräfte unbeschäftigt lassen und das Leben veröden“. 50

Anhand Baerwalds Glücksratgeber konnte bereits gezeigt werden, wie Anleitungen zum (Wieder-)erlangen einer „Arbeitsfreude“ auf der „suggestiven Macht“ einer Glücksgesinnung und diese wiederum auf der Grundlage arbeitspsychologischer Erkenntnisse basieren konnten. Im Folgenden werden anhand ausgewählter Schriften zum Thema „Arbeitsfreude“ aus der Arbeitswissenschaft und der Ratgeberliteratur der Wandel einer Politik von ,Glückskulturen“ untersucht. Gefragt wird: Welcher Zusammenhang bestand zwischen der kultur- und sozialpolitischen Frage nach der „Arbeitsfreude“ und der europaweit florierenden Glücksratgeberliteratur nach dem Ersten Weltkrieg?

\subsection{Ambivalentes Glückswissen in der Arbeitspsychologie}

Bereits vor dem Ersten Weltkrieg wurde die Psychotechnik 1912 vom deutschamerikanischen Arbeitspsychologen Hugo Münsterberg (1863-1916) in seinen Grundlagen einer Wirtschaftspsychologie ausgearbeitet. ${ }^{51}$ Der amerikanische Taylorismus (die Wissenschaft zur ,wirtschaftlichen Leistungsfähigkeit“"52) stand in der Tradition des technokratischen „Motor-Mensch“53-Modells. In Abgrenzung

49 Zur Frauenarbeit bei der Deutschen Post vgl. Nienhaus 1995. 1920, verhältnismäßig spät im Vergleich zu anderen deutschen Großbetrieben, wurde die „Psychotechnische Arbeitsgruppe Krupp“ mit einem „Psychotechnischen Institut“ gegründet. Dieses war an die Organisation der Lehrwerkstatt angeschlossen und hatte zur Aufgabe, die Arbeitsabläufe der Werktätigen an den Maschinen mit dem Ziel der Produktionssteigerung zu rationalisieren. Mehr dazu, vgl. Objektivität, Rationalität und Arbeitsplatzzufriedenheit. Die Psychotechnik im Unternehmen. In: PatzelMattern 2010, 124-252, 146.

50 Vgl. Baerwald 1933, 240.

51 Vgl. Münsterberg 1919.

52 Vgl. Rabinbach 1990a, 313.

53 Zum „Motor-Mensch“-Modell, vgl. Rabinbach 1990b, Gerovitch 2007, Technik und Ästhetik. Die visuelle Einstellung des Menschen. In: Stiegler 2016, 257-338. 
davon rückten Arbeitswissenschaftler`innen wie Münsterberg die physischen und vor allem psychischen Auswirkungen der Arbeit auf das Individuum in den Fokus. ${ }^{54}$ Münsterbergs Beobachtungen und Befragungen lenkten den Blick auf die betrieblichen Anforderungen und Rahmenbedingungen der Arbeitsprozesse. Damit verband Münsterberg angewandte Betriebspsychologie mit gesamtgesellschaftlichen Interessen. Das idealistisch überhöhte Ziel des Psychotechnikpioniers lautete:

\footnotetext{
„Die wirtschaftliche Experimentalpsychologie hat in der Tat vielleicht keine höhere Aufgabe, als diese Anpassung der Berufstätigkeit an die seelische Eigenart der Individuen, mit dem Ziel, das übervolle Maß seelischer Unbefriedigung an der Arbeit, seelischer Verkümmerung und Bedrücktheit und Entmutigung aus der Welt zu schaffen. ${ }^{\text {"55 }}$
}

Die angestrebte Wirkungsmacht der Psychotechnik als „Kulturaufgabe“56, wie sie Münsterberg beschrieb, beruhte noch bis in die 1920er Jahre auf der Annahme, mit Hilfe der psychotechnischen Wissenschaftlichkeit könne, neben der Reintegration der jungen Kriegssoldaten, nicht nur eine international wettbewerbsfähige Betriebsführung (durch Modernisierung der wirtschaftlichen Organisationsform und eine rationalisierte Arbeitsprozessplanung) herbeigeführt werden. ${ }^{57}$ Einige Arbeitspsycholog*innen, wie beispielsweise der Psychiater Wladimir Eliasberg (1887-1969), hofften, durch das Umstrukturieren des Mensch-Maschine-Modells mittels neuer Managementstrategien auch eine Steigerung der individuellen „Arbeitsfreude“ zu ermöglichen. Die Einführung von psychotechnischen Betriebsstellen mit ihren individuellen Berufseignungstests sah man als geeignete Praktik, den Erschöpfungserscheinungen entgegenwirken zu können. ${ }^{58}$ Als junge Fachdisziplin konnte die Psychotechnik flexibel auf die gesellschaftlichen Probleme der sich politisch, wirtschaftlich und gesellschaftlich wandelnden Weimarer Republik reagieren. Sie schien prädestiniert dafür zu sein, ihre unkon-

54 Zur europäischen, russischen und insbesondere deutschen Rezeption der amerikanischen Arbeitswissenschaft (z. B. Taylorismus, Fordismus) im deutschen Kaiserreich und in der Weimarer Republik vgl. u. a. Nolan 1994, Coenen u. a. 2010, Psychotechnik in Deutschland und Rußland. Der allgemeine Mensch. In: Stiegler 2016, 41-66.

55 Zukunftsentwicklung der Wirtschaftspsychologie. In: Münsterberg 1919, 174-183, 181.

56 Münsterberg 1920, 1.

57 Vgl. Normierung, Optimierung und Gestaltung. In: Patzel-Mattern 2010, 253-272, 255.

$58 \mathrm{Zu}$,sozialen Fragen in der Psychotechnik vgl. u.a Baumgarten 1931. Ein Beispiel aus Wien zeigt, wie im selben Zeitraum die Problematik der „Arbeitsfreude“ international diskutiert wurde, vgl. Kapitel zur „Arbeitsfreude“ (Baumgarten 1927c). 
ventionellen Methoden in den neu definierten Praxis- bzw. Untersuchungsfeldern zu testen. ${ }^{59}$

Nun könnte der Eindruck entstehen, dass Psychotechniker*innen an denselben sozialpolitischen Reformideen zur Verbesserung der Arbeitssituation und des individuellen Wohlbefindens der Arbeiterschaft interessiert gewesen wären wie die Glücksratgeber^innen jener Zeit. Baerwalds Vision eines „sozialen Zukunftsstaat $[\mathrm{s}]$ “60 demonstrierte dies zum Beispiel. Tatsächlich bestand in den unterschiedlichen Instituten weder bezüglich der sozialpolitischen Aufgabe der Psychotechnik noch der moralischen Verantwortung der Arbeitswissenschaftler*innen gegenüber der Gesellschaft ein Konsens. Vielmehr wurde ein ambivalenter Diskurs über Sinn und Zweck einer emotionspolitischen „Arbeitsfreude“ auf der Grundlage psychologischen Glückswissens geführt, was die folgenden Beispiel zeigen werden.

\section{4 „Der Kampf um die Arbeitsfreude“: Hendrik de Man und die NS-Arbeitspolitik „Kraft durch Freude“. Ein arbeitspsychologischer Vergleich}

„Der Kampf um die Arbeitsfreude“ betitelte der Arbeitswissenschaftler Hendrik de Man (1885-1953) ${ }^{61}$ programmatisch seine empirische Studie mit 78 Berichten von

59 Vgl. Normierung, Optimierung und Gestaltung. In: Patzel-Mattern 2010, 253-272, $254 \mathrm{ff}$. In ihrer methodischen Undifferenziertheit mangels empirischer Überprüfbarkeit mittels vergleichbarer längerer Zeiträume, ihrem ausschließlichen Fokus auf die Industriearbeit und ihrer Tendenz, vom Einzelfall universalistische Rückschlüsse qua Selbstüberschätzung zu ziehen, lagen Patzel-Mattern zufolge die Gründe für das Scheitern der Psychotechnik. Vgl. Normierung, Optimierung und Gestaltung. In: Patzel-Mattern 2010, 253-272, 258.

60 Vgl. Baerwald 1933, 316.

61 Hendrik de Man wurde am 17.11.1885 in Antwerpen geboren und starb am 20.6.1953 infolge eines Autounfalls in der Schweiz. Er studierte 1905-1909 in Leipzig Philosophie, Psychologie, Geschichte und Volkswirtschaft. In Leipzig kam er mit Karl Liebknecht, Karl Kautsky und Rosa Luxemburg in Kontakt. Als Journalist für unterschiedliche deutsche Zeitungen tätig, reiste er 1910 nach England, wo er mit der ansässigen Arbeiterbewegung vertraut wurde. Als Leiter einer Arbeiterbildungszentrale in Belgien kamen bei ihm angesichts des Ausbruchs des Ersten Weltkriegs Zweifel an der marxistischen Lehre auf. Nach zwei Jahren in den USA wurde ihm 1921 die Leitung einer Arbeiterhochschule in Belgien anvertraut. Von 1922 bis 1926 lebte er in Darmstadt und war in Frankfurt a. Main Dozent an der Akademie der Arbeit, parallel lebte er bis 1929 in Flims (Graubünden, Schweiz), von wo er an den Lehrstuhl für Sozialpsychologie der Universität Frankfurt berufen wurde. Seine wissenschaftlichen Arbeiten zeugen von einer Abkehr vom marxistischen Klassenkampfdenken hin zu einer sozialpsychologischen Beschäftigung mit der Arbeiterbewe- 
Industriearbeiter*innen und Angestellten. Darin gaben die Befragten Auskunft über ihre Gefühlserlebnisse bei der Arbeit. ${ }^{62}$ In einem Vergleich der arbeitspsychologischen Untersuchung von Hendrik de Man mit Robert Leys Propagandareden zur NS-Arbeitspolitik „Kraft durch Freude“ und mit Carl Arnholds (18841970) arbeitswissenschaftlichen Studien zur „Arbeitsfreude“ soll im vorliegenden Kapitel aufgezeigt werden, inwiefern die ausgewählten Schriften neben Brüchen auch ambivalente Kontinuitäten von arbeitspsychologischem Glückswissen aufweisen. Gefragt wird: Wie definierte der Arbeitspsychologe de Man nach der empirischen Auswertung seiner Studienergebnisse „die Arbeitsfreude“ eines Individuums im gesellschaftspolitischen Kontext der Weimarer Republik im Vergleich zur NS-Arbeitsmoral „Kraft durch Freude“?

In seiner empirischen Studie zur „Arbeitsfreude“ befragte Hendrik de Man seine Studierenden, welche an der „Akademie der Arbeit“ in Frankfurt a. Main zwischen 1924 und 1926 die zweisemestrige Vorlesung „Psychologie des Industriearbeiters“ belegt hatten. Anhand eines Fragebogens sollten sie ihre „Gefühle auf der Arbeit“ in Bezug auf „Arbeitsfreude“ und „Arbeitsunlust“ kritisch reflektieren und darüber einen Bericht verfassen. ${ }^{63}$ Wie viele Fragen die Angestellten beantworteten, stand ihnen frei. Gefragt wurde beispielsweise:

„[...] Von welchen Gefühlen sind Sie beherrscht [...] gegenüber:

a) den von Ihnen benutzten sachlichen Arbeitsmitteln (Werkzeug, Maschinen, Betriebsausstattung)?

b) den Arbeitskollegen im Betrieb?

c) dem Vorgesetzten im Betrieb?

d) Ihrer Arbeitstätigkeit überhaupt?

e) Ihren gewohnten Tätigkeiten außerhalb des Betriebs? [...]

gung, dem Sozialismus und Faschismus unter Berücksichtigung sozialhistorischer Wandlungsprozesse (z.B in „Idee des Sozialismus“ 1933). Nach 1933 wurde ihm die Lehrtätigkeit untersagt. In Belgien entwickelte er als sozialistischer Politiker den „Plan der Arbeit“ zur Überwindung der Wirtschaftskrise und übernahm das Ministeramt für öffentliche Arbeit, seit 1936 das Ministeramt für Finanzen. 1939 wurde er Vorsitzender der Belgischen Arbeiterpartei. Im Januar 1940 trat er aus der Regierung aus, nachdem er sich für die Neutralitätspolitik eingesetzt hatte. Mit dem Einmarsch der deutschen Truppen am 10.5.1940 empfahl er als Berater des Königs Leopold II. die Kooperation. Unter dem NS-Regime erfüllte er überparteiliche Aufgaben und schrieb für seine Zeitung „Le Travail“ (1941-1942). 1941 erhielt er ein Rede- und Publikationsverbot und zog sich Ende 1941 bis August 1944 nach Savoyen zurück. Als politischer Flüchtling lebte er bis zu seinem Tod 1953 in der Schweiz und war als Schriftsteller tätig. 1946 wurde er von einem belgischen Gericht wegen Kollaboration zu einer 20-jährigen Gefängnisstrafe verurteilt und verlor seine Staatsbürgerschaft. Vgl. Krause 1990.

62 Vgl. Man 1927, 4 f.

63 Vgl. ebd., 3. 
1. Besteht nach Ihrer eigenen Erfahrung ein Zusammenhang zwischen Arbeitsfreude und a) der Einrichtung der Betriebsräte, b) der Tätigkeit der gewerkschaftlichen Organisation, c) der allgemeinen sozialen Einstellung des einzelnen Arbeiters?

2. Halten Sie in Ihrem Beruf einen höheren Grad von Arbeitsfreude für möglich, und wenn ja, auf Grund welcher Veränderungen

a) der Arbeitstechnik [...]

b) der Arbeitsorganisation im Betriebe?

c) der gesellschaftlichen Organisation überhaupt? [...]“64

Welches Glückswissen über die „Arbeitsfreude“ konnte de Man aus dem empirischen Material gewinnen? Im Schnitt gaben 57\% aller Befragten über ihre „Freude“ bei der Arbeit eine Plusaussage (,+“) von positiven Momenten an, 24\% ein +/- und 19\% ein „-““. ${ }^{65}$ Zur Interpretation dieser statistischen Werte betont de Man die Ambivalenz, welche ihnen zugrunde liege. Aus den Berichten gehe hervor, dass jede Person, die grundsätzlich „Arbeitsfreude“ empfände, auch „unlusterzeugende“"66 Momente kenne. Umgekehrt empfinde derjenige, der seine Arbeit als anstrengend beschrieb, gelegentlich auch „arbeitsfreudige Momente“ ${ }^{67}$ Obwohl die nichtrepräsentativen Aussagen keine allgemeingültigen Schlüsse zuließen, kommt de Man nach Durchsicht der Berichte zu folgendem Zwischenfazit: „Nach Arbeitsfreude strebt jeder Arbeitende, wie jeder Mensch nach Glück strebt. Arbeitsfreude verlangt gar nicht danach, ,gefördert' zu werden; es kommt nur darauf an, daß sie nicht gehemmt wird." "68 Ausgehend von der zunächst trivial anmutenden Feststellung, dass die Arbeiterschaft unabhängig von materiellen oder finanziellen Anreizen grundsätzlich nach einem „Glück“ der „Arbeitsfreude“ strebe, fragt de Mans Studie nach den psychologischen Faktoren, welche als „fördernde“ und „hemmende“ Motive auf die Arbeitsfreude einwirken. Dabei entwirft de Man eine schematische Triebtheorie, die von einer Spannung zwischen dem „Drang zur Arbeitsfreude“69 und deren „Hemmungen“ gekennzeichnet sei. $^{70} \mathrm{Zu}$ dieser streitbaren Theorie gelangte de Man über die Analyse der Erfah-

64 Ebd., 4.

65 Vgl. ebd., 147.

66 Ebd., 148.

67 Vgl. ebd., 147.

68 Ebd., 148.

69 Zum Schema vgl. ebd., $149 \mathrm{f}$.

70 De Man unterscheidet in seinem Schema zwischen „elementar-triebhafte[n] Motiven“ wie „Tätigkeitstrieb“, „Spieltrieb“, „Aufbautrieb“, „Erkenntnistrieb“, „Geltungstrieb“ und „Besitztrieb“, die den „Drang zur Arbeitsfreude“ fördern, sowie „gelegentlich fördernde[n] Motive[n]“ wie „Herdentrieb“, „Herrschsucht und Unterordnungsverlangen“, „ästhetische Befriedigung“, „Erwägungen des Privatvorteils“ und des „,sozialen Nutzens“. Als „Hemmungen“ bezeichnet er 
rungsberichte. Er verglich deren Aussagen und erläuterte systematisch die psychologischen Probleme und sozialpolitischen Einflüsse im Berufsalltag.

Aus der Analyse der in de Mans Studie abgedruckten Erfahrungsberichte gehen folgende Erkenntnisse hervor: Ein entscheidendes Motiv der „Arbeitsfreude“ sei die Gewissheit der Arbeiter*innen darüber, dass ihr Tageswerk nicht nur als existentieller Brotverdienst angesehen würde. Wenn die Tätigkeit Eigeninitiative verlangte, sich einigermaßen abwechslungsreich gestaltete und in Relation zu außerbetrieblichen Aktivitäten stand (z. B. gewerkschaftlichem Engagement), dann konnte die Arbeit in einen größeren gesamtgesellschaftlichen Kontext gestellt und dadurch in ihrer Entbehrung relativiert werden. So formuliert es beispielsweise ein 35-jähriger angelernter Spinnweber:

„Der Spinnerberuf verlangt Initiative. Die Spinnerei ist eine rege unterschiedliche Arbeit. [...] Es gibt in der Spinnerei ein Hasten und Schuften und ein bequemes Arbeiten Faulenzen. Bei der Bedienung meiner Maschine war ich von angenehmen Gefühlen beherrscht, weil mir die Arbeit vollständig Zeit ließ, meine politischen und gewerkschaftlichen Vorträge auszuarbeiten. Ein automatischer Blick genügte, um die 2000 Fäden zu überschauen. “71

Ein 28-jähriger gelernter Buchbinder nennt noch zwei weitere Faktoren, nämlich die „tarifliche Regelung des Wochenlohnes und der Ferien“, welche ihm ein „Gefühl der Sicherheit“ verliehen. ${ }^{72}$ Positiv wirkten sich außerdem die flache Betriebshierarchie und die Kollegialität unter den Mitarbeiter*innen auf seine „Arbeitsfreude“ aus: „Betriebshierarchie besteht nicht, im Gegenteil, es herrscht selbst zwischen Vorgesetzten und Kollegenschaft ein durchaus kameradschaftlicher Geist.“73

Ein 40-jähriger Lokomotivfahrer betont, dass das Wissen um die Existenz der gewerkschaftlichen Organisation und die Repräsentation der Arbeiterschaft im Betriebsrat seine „Dienstfreudigkeit“ begünstigten: „Für mich als Person ließ der Gedanke, gewerkschaftlich organisiert zu sein und in den Betriebsräten eine wesentliche Stütze zu haben, jederzeit das Gefühl der Dienstfreudigkeit aufkommen"74.

erstens „arbeitstechnische Hemmungen“, zweitens „innerbetriebliche soziale Hemmungen“ sowie „außerbetriebliche soziale Hemmungen“. Mit Blick auf die Forschungsfrage würde es an dieser Stelle zu weit führen auf die einzelnen Theorieaspekte einzugehen. Zum Schema, vgl. Man $1927,149 \mathrm{f}$.

71 „Fall 24“, m, 35, gelernter Spinnweber. Vgl. Man 1927, 36.

72 „Fall 68“, m, 28, gelernter Buchbinder. Vgl. Man 1927, $96 \mathrm{f}$.

73 „Fall 68“, m, 28, gelernter Buchbinder. Vgl. Man 1927, $96 \mathrm{f}$.

74 „Fall 76“, m, 40, Lokomotivfahrer, gelernter Schmied, Schlosser. Vgl. Man 1927, 111. 
Ein Mindestmaß an Selbstverantwortung und gewerkschaftlichem Engagement forderte auch eine 25-jährige gelernte Stenotypistin, die in einem Pressebüro als Sekretärin, Buchhalterin und Kontoristin arbeitete. „Arbeitsfreude“ empfand sie eigentlich nur, wenn sie für sich „persönlich“ etwas schaffen konnte, womit sie politische und gewerkschaftliche Aktivitäten meinte, die in der Regel ein unerlaubtes inoffizielles Tun bedeuteten, das „zu Hast und Gewissenskonflikten“ drängte. ${ }^{75}$ Trotz unliebsamer Fleißarbeit empfand sie immer dort „Arbeitsfreude“, „[...] wo diese [Arbeit] eine größere geistige Anspannung erfordert, wo immer eine gewisse Aufregung herrscht [...] eine gewisse Auffassungsgabe und eine gewisse politische Beflissenheit erfordert" ${ }^{\star 76}$.

Eine 25-jährige gelernte Kontoristin, die als Stenotypistin in der technischen Abteilung einer städtischen Bibliothek arbeitete, nennt weitere Umstände, die ihre „Arbeitsfreude“ erhöhten: Zunächst seien es die arbeitsfreundlichen Räumlichkeiten der Bibliothek, die lichtdurchflutet und ordentlich waren und es erlaubten, den Arbeitsplatz individuell zu dekorieren. In dieser subjektiven Gefühlsbeschreibung kommt die emotionshistorische Bedeutung des Arbeitsorts als individueller Gefühlsraum zum Ausdruck, bei dem architektonische und selbstgestalterische Gefühlselemente sich günstig auf die individuelle „Arbeitsfreude“ auswirkten. ${ }^{77}$ Weiter nennt die Kontoristin das „ausgezeichnete persönliche Verhältnis zwischen mir als ,technischer Kraft" und den mir überstellten Bibliothekaren und wissenschaftlichen Fachreferenten, mit denen ich zusammenarbeiten musste. Die Anerkennung auch der nicht ,rein geistigen' Arbeit und des Menschen, der sie leistet, steigerte in der Tat die Arbeitsfreude. "78 Sie betont das soziale Gemeinschaftsgefühl innerhalb der Belegschaft, welches im Idealfall durch einen unhierarchischen Umgang und eine kommunikative Zusammenarbeit von der technischen Angestellten bis zur wissenschaftlichen Fachreferentin ein Gemeinschaftsgefühl vermittelt. Heutzutage bezeichnet das psychologische Wissensmanagement solche Fälle als „prosoziales Verhalten“ bei der Arbeit. Diese fachliche Begriffsentwicklung in der angewandten Arbeitspsychologie stützt die These der kontinuierlichen, wechselseitigen und transnationalen Wissenszirkulation über „Arbeit“ und „Glück“.79

75 „Fall 18“, w, 25, angelernte Stenotypistin. Vgl. Man 1927, 29.

76 „Fall 18“, w, 25, angelernte Stenotypistin. Vgl. Man 1927, 29.

77 Mehr zum gefühlshistorischen Konzept der „Gefühlsräume“ vgl. u.a. Gammerl und Herrn 2015.

78 „Fall 31“, w, 25, gelernte Kontoristin. Vgl. Man 1927, 50.

79 „Prosoziales Verhalten“ oder „Prosozialität“ hat im Wesentlichen drei soziale Voraussetzungen zu erfüllen: Empathie als soziale Bindung, Bereitschaft zur Perspektivenübernahme sowie ein befriedigendes Kosten-Nutzen-Verhältnis. Prosoziales Verhalten trägt zur Unterstützung anderer 
Ein weiteres Element der „Arbeitsfreude“ ging über den Betriebsalltag hinaus und betraf die Selbstverwirklichung des individuellen Lebensstils der Kontoristin und das Verlangen, sich weiterzubilden und sich mit Gleichgesinnten auszutauschen. ${ }^{80}$ Aus diesem Grund schätzte die Kontoristin die Möglichkeit, sich bei Vorträgen oder beim Lesen der Bibliotheksbücher neues Wissen anzueignen und im Kreis der Mitarbeiter`innen „Fragen der Jugendbewegung“ diskutieren zu können. ${ }^{81}$ In selbstorganisierten Lesekreisen konnte sie „das Bedürfnis nach eigener Charakterisierung gelesener Bücher“ zum Ausdruck bringen und den „Drang nach noch größerer geistiger Arbeit und Anregung, als die Arbeit selber bot" stillen. ${ }^{82}$

Eine 27-jährige „ungelernt[e]“ Zigarrenmacherin, die zahlreiche Berufswechsel durchlebt hatte, benennt, neben der Freiheit politisch in der SPD (Arbeiterjugend, Jungsozialistengruppe, Frauengruppe) und gewerkschaftlich (Fabrikarbeiterverband, Kassiererin und Verwaltungsmitglied) aktiv $\mathrm{zu}$ sein, einen musikästhetischen Aspekt, der ihr und ihren Kolleg*innen trotz Ermüdungserscheinungen „Arbeitsfreude“ verschaffe:

„Sehr oft, und fast immer nach der Mittagspause, verlangten die Kolleginnen Gesang im Raum, um nicht einschlafen zu müssen. In dieser Beziehung herrscht in einer Zigarrenfabrik vollständige Freiheit, und ich habe die Empfindung, daß durch den Gesang auch die Eintönigkeit und Langeweile unterdrückt worden ist, ja sogar die Produktivität gesteigert wurde. “83

De Man zeigte sich vom Selbstbewusstsein und der „unbändigen Natur“ dieser jungen Frau beeindruckt. ${ }^{84}$ Zunächst kündigte sie eine Stelle als Hausangestellte bei einem Regierungsrat in Berlin, weil man ihr das Zeitungslesen verboten hatte. Danach schlug sie einen Karrieresprung zur Meisterin in einer Filmfabrik aus, weil ihr im Gegenzug dazu die gewerkschaftliche Tätigkeit untersagt worden wäre: „Auf ihr Aussehen und Benehmen hin würde sie jede dienstbotensuchende „Herrschaft‘ für eine ,Perle‘ halten: still, freundlich, zurückhaltend fast bis zur Schüchternheit, dabei zweifellos gewissenhaft und arbeitsfreudig“, so de Man. ${ }^{85}$

Personen auf der Arbeit bei und erfolgt außerhalb des Pflichtenhefts und wird nicht materiell vergütet. Vgl. Moser 2002.

80 „Fall 31“, w, 25, gelernte Kontoristin. Vgl. Man 1927, 50.

81 „Fall 31“, w, 25, gelernte Kontoristin. Vgl. Man 1927, 50.

82 „Fall 31“, w, 25, gelernte Kontoristin. Vgl. Man 1927, 50.

83 „Fall 7“, w, 27, „, ungelernte Zigarrenmacherin“. Vgl. Man 1927, $16 \mathrm{f}$.

84 Vgl. Man 1927, 17.

85 Vgl. ebd. 
An diesem Beispiel lässt sich aus einer gefühls- und wissenshistorischen Perspektive aufzeigen, welche stereotypisierten Verhaltensnormen für eine weibliche Dienstbotenstelle aus Sicht des (männlichen) Psychologen als geeignet erschienen: Freundlichkeit, Zurückhaltung, Gewissenhaftigkeit und „Arbeitsfreude“ waren an geschlechterstereotype Eigenschaften geknüpft. In der Konsequenz stellten diese von gesellschaftlichen Konventionen geprägten, normativen Charaktereigenschaften hemmende statt fördernde Motive für die „Arbeitsfreude“ der jungen, sich emanzipierenden Frau dar. Dieser geschlechterpolitische Aspekt des „Kampf[es] um die Arbeitsfreude“, der mit den drängenden Fragen nach weiblicher Persönlichkeitsentfaltung und Berufsverwirklichung in der Weimarer Republik einherging, kam in de Mans Untersuchung nur am Rande zur Sprache. Ein Grund dafür mag das repräsentative Ungleichgewicht von nur 5 weiblichen im Gegensatz zu 72 männlichen Befragten gewesen sein. Dies wiederum gibt einen geschlechterhistorischen Einblick in das Auswahlverfahren der Gewerkschaftsverbände für die Zulassung der Hörerschaft an der Akademie der Arbeit in Frankfurt a. Main.

Inwieweit die NS-Arbeitswissenschaft auf die Forderungen der jungen Frauen nach Selbstbestimmung und freier Berufswahl eingingen, wird im 13. und 14. Kapitel aufgezeigt. Exemplarisch seien hier die NS-Parteiorganisationen wie „Bund deutscher Mädel“, „NS-Arbeitsdienst“ oder die Freizeitorganisation „Kraft durch Freude“ ${ }^{86}$ genannt.

Wie lässt sich das Glückswissen, welches de Man als empirische Erkenntnisse in seiner Studie präsentiert, zusammenfassen? Entscheidende „Motive“ hätten einerseits mit außerbetrieblichen Aktivitäten der Arbeiter`innen („Familienleben, die Schwänke, das Kino, der ,Sport‘, die Erotik, das Glücksspiel, die Vereinsmeierei [...]“), andererseits mit den „innerbetrieblichen Wirkungen des Betriebskomplexes“ zu tun. Damit meint de Man insbesondere die repetitive Teilarbeit der Rationalisierung, in welcher er „die Hauptursache [der] seelische[n] Loslösung des Arbeiters von seiner Arbeit“ sieht. $^{87}$

86 Vgl. Ley 1937b.

87 Vgl. Man 1927, 116. Zu den „technischen Hemmungen“ gehören, neben schlechten technischen Betriebszuständen, eine „Einseitigkeit der Bewegung“, Verringerung der eigenen Arbeitsinitiative und Aufmerksamkeit sowie hypnotische Rhythmisierung, die zu Ermüdungserscheinungen führen. Als „innerbetriebliche soziale Hemmungen“ nennt de Man die in sozialen Arbeitsbedingungen zu verortenden ungerechten Lohnsysteme. Darunter fallen ein Arbeitsleistungszwang, eine „Überbevorteilung zugunsten des Unternehmens“, „Gefährdung der Solidarität“ unter den Arbeitenden und der „Arbeitsqualität“, „Bedrohung der Existenzsicherheit“, „Unkontrollierbarkeit der Leistungs- und Lohnhöhe“ sowie eine ausgeprägte „Betriebshierarchie“. Vgl. Man 1927., 149f. und $290 \mathrm{f}$. 
Als „gelegentlich fördernde Motive“ nennt de Man pejorativ einen „Herdentrieb“, worunter der Psychologe die „Geselligkeit“ und das sozialpolitische Gemeinschaftsgefühl der oft gewerkschaftlich aktiven Belegschaft im Großbetrieb versteht. ${ }^{88}$ Die Komplementärerscheinung aus „Herrschsucht“ (oder „Autoritätssucht“) und einer „Freude an der Unterordnung“ können nach de Mans Einschätzung, die erneut mit kritischer Distanz zu beurteilen ist, positive Auswirkungen auf die „Arbeitsfreude“ haben. ${ }^{89}$ Hinzu kommen Aspekte der „ästhetische[n] Befriedigung“, „rationale[r] Erwägung des Privatvorteils“ und des „Sozialen Nutzens“. ${ }^{90}$

Es stellt sich die Frage, inwiefern die Resultate der Studie zur „Arbeitsfreude“ in der Weimarer Republik repräsentativ für die Gefühlswelt der Arbeiterschaft waren. Nach vollständiger Sichtung der abgegebenen Berichte entschied sich de Man 1927 für eine Publikation, da ,in der Öffentlichkeit genug Interesse vorhanden sein dürfte - oder sollte “. ${ }^{91}$ Bei den Befragten handelt es sich de Mans eigener kritischer Einschätzung zufolge um eine Selektion einer „bildungshungrige[n] Elite der jüngeren Arbeitsgeneration“, die jährlich von den Gewerkschaftsverbänden als Hörer*innen ausgewählt wurde. Die Mehrheit der Befragten war zwischen 20 und 30 Jahre alt und hatte schon einige Jahre Berufserfahrung gesammelt. Viele von ihnen waren entweder politisch und/oder gewerkschaftlich aktiv. Die Frage nach der Repräsentativität sei beim Versuch, verallgemeinernde Schlussfolgerungen ziehen zu wollen, unvermeidlich, so der Autor selbstkritisch.

Ein Blick in das Nachbarland Schweiz kann auf die kritische Frage nach der Repräsentativität einen Anhaltspunkt bieten. Wie wurde de Mans Studie in der Schweiz rezipiert und wie wurden seine arbeitspsychologischen Befunde beurteilt? Der Schweizer Max Weber (1897-1974), gewerkschaftlicher Wirtschaftspolitiker, späterer SP-Bundesrat (1951-1953) und Dozent für Volkswirtschaft und Finanzpolitik an den Universitäten Bern (1948-1951, 1956-1966) und Basel

88 Vgl. ebd., 168f. Zum Vergleich: Bereits Münsterberg wies in seiner Grundlagenforschung zur Wirtschaftspsychologie im Kapitel zur „Gewinnung der bestmöglichen Leistungen“ neben Störfaktoren der Aufmerksamkeit, dem Problem der „Monotonie“ und „Ermüdung“ auf „[p]hysische und soziale Einflüsse auf die Leistungsfähigkeit“ hin. Vgl. Münsterberg 1919, 86-142.

89 Vgl. Man 1927, 169-172.

90 Vgl. ebd., 150, 168-176. Zu den außerbetrieblich sozial „hemmenden“ Gefühlen der „Arbeitslust“ zählen das „Gefühl der chronischen Angst und der Machtlosigkeit“, der „Existenzunsicherheit“, der „gesellschaftliche(n) Inferiorität“, was sich in sozialer Aggressivität gegenüber dem Vorgesetzten ausdrücken könne, das fehlende Identifikationsgefühl mit der eigenen Tätigkeit und dem Betrieb, und die „Geringschätzung der Arbeit“ durch den Arbeitsgeber. Vgl. Man 1927, $283 \mathrm{ff} ., 280-289$.

91 Vgl. Man 1927, 3. Einige Befragungsergebnisse hatte de Man bereits in die Studie „Zur Psychologie des Sozialismus“ (1926) einfließen lassen. 
(1954-1966), verfasste 1929 in der „Gewerkschaftlichen Rundschau für die Schweiz" eine Rezension über de Mans Studie. ${ }^{92}$ Weber gab zwar zu Bedenken, dass die Hörer^innen womöglich sowohl vom Fragebogenschema als auch vom vermittelten Vorlesungswissen „suggestiv“ beeinflusst worden seien. Nichtsdestotrotz schätzte Weber de Mans Untersuchung als „bahnbrechende Arbeit“ ein. ${ }^{93}$ Für seine Beurteilung stützte sich Weber auf seine eigene empirische Studie, die er - angeregt durch de Mans Arbeit - in einem Ferienkurs zur Psychotechnik an der Schweizerischen „Arbeiterbildungszentrale“ durchgeführt hatte. „Es ist direkt auffallend, wie weitgehend die Meinungsäusserungen unserer Ferienkursteilnehmer die Ergebnisse der Untersuchung de Mans bestätigen. Daraus darf man wohl schliessen, dass es sich in den beiden Fällen um symptomatische Aeusserungen handelt“, schlussfolgert Weber. ${ }^{94}$ Gleichzeitig räumt der Wirtschaftspolitiker ein, dass es sich bei seinen Befragten um eine noch weniger repräsentative Zahl gehandelt habe als bei de Man. Bedauerlicherweise sei de Mans Studie noch zu wenig von der wissenschaftlichen Psychologie beachtet worden. Obwohl sich die Psychotechnik mit „Arbeitsfreude“ befasse, tue sie dies noch „zu sehr bloss unter dem Gesichtspunkt der Leistungssteigerung“, so der spätere SP-Bundesrat. ${ }^{95}$

Eine der wichtigsten Resultate aus der Umfrage war für de Man die Erkenntnis, dass die „Möglichkeit zum höchsten Glück“ darin bestehe, „für das Glück anderer zu wirken“. ${ }^{96}$ Die „Lösung für das Problem der Arbeitsfreude“ sieht der Sozialpsychologe folglich in der Schaffung eines „neuen Arbeitsethos“ mit einer „sittlichen Pflicht“, deren „Grundlage die Idee der Arbeitspflicht als Schuld an die Gemeinschaft“ sei. ${ }^{97}$ Im selbstgewählten, emotionspolitischen Korsett eines „sozialen Pflichtgefühls“, das wirksamer sei als die „sozialistische Ideologie des Gemeinwohls“ oder die „kirchliche Ideologie der Arbeitspflicht“, ist de Man

92 Vgl. Weber 1929.

93 Vgl. ebd., 291.

94 Vgl. ebd., 293.

95 Vgl. Weber 1929, 290. Zum Stand der Arbeitswissenschaft und Psychotechnik in der Schweiz vgl. Eintrag im Handwörterbuch der Arbeitswissenschaft von Prof. Dr. Jules [Julius] Suter (1882-1959), Universität Zürich, 1923 Gründer des Psychotechnischen Instituts, vgl. Suter 1930. Zur Person, vgl. https://www.paedagogik-feiert.uzh.ch/de/personenbiographien/suterjules. html, 13.08.2020.

96 Vgl. Man 1927, 289.

97 Vgl. ebd., 183. 
überzeugt, wirke sich die Arbeitspflicht ,in einer Schicksalsgemeinschaft aus, die alle Arbeiter ohne Unterschied des Glaubensbekenntnisses umfaßt. “98

An diesem Beispiel kann die rezeptionshistorische Kontinuität von arbeitspsychologischem Glückswissen in der NS-Arbeitsmoral „Kraft durch Freude“, der Betriebspropaganda der „Deutschen Arbeitsfront“ und der arbeitswissenschaftlichen Forschung bei Carl Arnhold aufgezeigt werden.

Folgender Ausschnitt aus Robert Leys Gründungsrede der Freizeitorganisation „Kraft durch Freude“ von 1933 zeigt, in welchem Ausmaß NS-Funktionäre wie Ley und Carl Arnhold ihre Gefühlspropaganda auf de Mans arbeitspsychologischem Glückswissen aufbauten:

„In Belgien versuchte ein Dr. de Man etwas tiefer in diese Probleme einzudringen. Er stellte die These auf, daß man in der Freizeit die Persönlichkeit heben und die Minderwertigkeitskomplexe beim Arbeiter beseitigen muß. Wir sehen hier Ideen, die auch unsere Gedanken in unserem Werk grundsätzlich beeinflussen.“99

Zur wissenshistorischen Untersuchung einer Kontinuität von arbeitswissenschaftlichem Glückswissen ist auch die Rede Robert Leys über „De[n] deutsche[n] Mensch[en] und seine Lebensgestaltung“ aufschlussreich:

\begin{abstract}
„Der Takt der Maschine muss mit dem Rhythmus des Blutes in Einklang gebracht werden. Hier lag die völlig verkehrte und falsche Auffassung unserer Wirtschaftsführer in den Nachkriegsjahren. Man glaubte, mit amerikanischen Arbeitsmethoden die unter dem Schlagwort ,Rationalisierung kalt und nüchtern die Arbeitsmethoden nach dem Takt der Maschine errechneten, als dem krassesten Ausdruck wirtschaftlicher Rentabilität, richtig zu verfahren, und man mußte dann erfahren, daß diese für die deutsche Rasse völlig unangebrachten Methoden nicht nur nicht wirtschaftlich waren, sondern im Gegenteil zum Schaden des Unternehmens ausschlugen.

Der Mensch darf nie überlastet, aber ebenso müssen er und seine Fähigkeiten voll ausgenutzt werden. Dann allein wird ihm die Arbeit zur Freude. Urlaub, Erholung, schöne Arbeitsplätze, Freizeitgestaltung, alles das dient letzten Endes nicht allein dem Arbeiter, sondern dem Unternehmer zu gleichen Maßen. Was nützt dem Unternehmer ein übermüdeter und nervöser Mensch?“100
\end{abstract}

98 Vgl. ebd., 182. Der Vergleich mit der Definition zur Arbeitsethik im offiziellen „Handwörterbuch der Arbeitswissenschaft“ in Kap. 10.5 wird Aufschluss über die wissenshistorische Bedeutung von de Mans Arbeitsethik im Kontext der NS-Arbeitsideologie geben.

99 Ley 1935, 29. Denkanstoß zu dieser These gab Sascha Howinds Kapitel über de Man, vgl. Howind 2013, $84 \mathrm{ff}$.

100 Ley 1936a, $50 \mathrm{f}$. 
An diesen Beispielen kann gezeigt werden, wie intensiv sich Ley mit dem Wissen über leistungsorientierte Rationalisierung und den arbeitspsychologischen Erkenntnissen über „Arbeitsfreude“ auseinandergesetzt hat. Transnational vergleicht er die arbeitswissenschaftlichen Studien mit den gewerkschaftlich oder staatlich betriebenen Freizeitorganisationen in Russland, Frankreich, Belgien und im Besonderen mit „Dopolavoro-Italien“101 und wägt deren Umsetzungserfolge im propagandistischen Abgleich mit der NS-Arbeitsmoral „Kraft durch Freude“ ab.

Das Paradoxe an Leys emotionspolitischer Rhetorik war, dass er das ,alte Psychowissen des rationalisierten Mensch-Maschine-Modells in dessen angeblich arbeitsideologischem Verzicht auf einen Anspruch individueller „Arbeitsfreude“ kritisierte. Zugleich instrumentalisierte er die „Arbeitsfreude“ einerseits im Interesse eines imaginierten Arbeiterkollektivs „einer deutsche[n] Rasse“, andererseits im wirtschaftskapitalistischen Interesse der „Unternehmer“. Leys Ziel war es, den Begriff der „Arbeitsfreude“ in Einklang mit der rassistischen NS-Arbeitsideologie und den wirtschaftskapitalistischen Interessen des totalitären NS-Regimes zu bringen. Schon 1931 hatte Hitler in einem Gespräch mit dem Gewerkschafter August Winnig auf die zukünftige Wirtschaftssynergie aus Nationalsozialismus und Kapitalismus unter Beihilfe „zweckmäßig ausgebildete[r] Wirtschaftsmethoden“ insistiert, wozu das „rassenhygienisch“ umgedeutete Wissen der arbeitswissenschaftlichen Psychotechnik zählen dürfte. ${ }^{102}$

Ley hoffte im Rückgriff auf das arbeitspsychologische Glückswissen über eine autosuggestive Willenskraftideologie („Kraft durch Freude“) die wirtschaftspolitische und militärische Strategie der industriellen Produktionssteigerung legitimieren zu können. Welche Bedeutung der arbeitspsychologischen Gefühlspolitik im Rahmen der NS-Arbeitspsychologie zukam, wird im Dialog zwischen Adolf Hitler und Robert Ley, dem Mitbegründer der „NS-Gemeinschaft ,Kraft durch Freude““ und Leiter der „Deutschen Arbeitsfront“, über die emotionspolitische Bedeutung von „Kraft durch Freude“ in Leys Rede „Der Glaube des deutschen Arbeiters verpflichtet!“ deutlich:

101 Zum transnationalen Vergleich bei Ley, vgl. Ley 1940, $28 \mathrm{ff}$.

102, „[...] Kapitalismus als historische Erscheinung [...]. [Hitler war laut Winnig der Auffassung], dass es Unsinn sei, den Kapitalismus abschaffen zu wollen, sinnvoll dagegen, das Beste für den Staat aus ihm herauszuholen und ihn dort, wo er den Anforderungen des Staates nicht genügte, durch zweckmäßig ausgebildete Wirtschaftsmethoden zu ersetzen; alsdann möge sich aus diesen Ersatzstücken einmal ein neues Wirtschaftssystem ergeben und bilden - das sei dann eine neue gewachsene Ordnung, die ihrerseits eine neue Wirtschaftsepoche einleite.“ Winnig 1946, 39. 


\begin{abstract}
„,Kraft durch Freude، ist vielleicht die kürzeste Formel, auf die ich das nationalsozialistische Wollen überhaupt bringen kann. Der Führer sagte kürzlich einmal zu mir: ,Ley, Sie haben recht, alles kommt letzten Endes aus der Freude. Wie sind Sie überhaupt auf diesen Namen gekommen? Es ist wirklich richtig: Kraft durch Freude. Wir wollen, daß unser Volk kräftig durch Freude werde, damit Deutschland ewig werde.““103
\end{abstract}

Ley glaubte eine Handlungslegitimation aus dem empirischen Glückswissen der arbeitspsychologischen Forschung, wie jener de Mans, ableiten zu können. Dieser rhetorische Legitimationsversuch war im totalitären NS-Regime ein de facto überflüssiges diplomatisches Manöver gegenüber den ehemaligen Wähler`innen, welches auf einem demokratischen Rechtsstaatlichkeitsverständnis aus der Weimarer Republik basierte. Im „Dritten Reich“ hatte diese Rhetorik nunmehr eine rein machtpolitische Stabilisierungsfunktion, indem sie der Propagandastrategie, die Menschen emotionspolitisch an das neue Regime zu binden, diente. Wie war eine solche diskursive Kontinuität von arbeitspsychologischem Glückswissen angesichts der radikalen sozialpolitischen Umbrüche nach 1933, welche die „Gleichschaltung“ und „Arisierung“ der Betriebe, „rassenpolitische“ Verfolgungen, Massenverhaftungen und Exil mit sich brachten, zu erklären?

De Man hatte bereits 1927 argumentiert, dass sich „historisch“ gezeigt hätte, dass eine Reduktion der Arbeitszeit und eine Lohnsteigerung die „Arbeitsfreude“ nicht steigere, sondern nur ein massenpsychologisches Konsumbedürfnis mittels außerbetrieblicher Zerstreuungen geweckt hätte, welches „Arbeitsunlust“ hervorriefe:

„Die Richtung der Massenbedürfnisse auf die Lebenssphäre außerhalb der Arbeit ist, historisch gesehen, nicht die Ursache, sondern die Folge eines Verlustes an Arbeitsfreude [...]. Sie bedeutet demnach nichts anderes als eine Ablenkung des Dranges zum Glück von einem versperrten Gebiet nach einem anderen, leichter zugänglichen. [...] Das Glück, das man in der Arbeit nicht mehr fand, suchte man außerhalb.“104

In Leys propagandistischer Umdeutung dieses arbeitspsychologischen Glückswissens lautete die Schlussfolgerung im Sinne der menschenverachtenden, totalitären NS-Arbeitsmoral:

„Wir Nationalsozialisten sehen das Arbeitsverhältnis, die Arbeitsmethoden, die Arbeitsbedingungen, die Arbeitsentwicklung und daraus eine ständige Leistungssteigerung, die eine natürliche Erhöhung des Einkommens der Menschen bedingt. Nicht die Hebung des

103 Ley 1939, 274.

104 Man 1927, $226 \mathrm{f}$. 
Lohnniveaus der Menschen muß erreicht werden. Für uns steht der deutsche Mensch im Mittelpunkt unseres Wollens." ${ }^{\text {105 }}$

Die NS-ideologische Umdeutung von de Mans arbeitspsychologischem Wissen über das angeblich nicht vorhandene Abhängigkeitsverhältnis zwischen „Arbeitsfreude" und Lohnerhöhung lautete im nationalsozialistischen Entwurf eines „deutschen Betriebswesens“ des Arbeitswissenschaftlers Carl Arnhold, Gründer des „Deutschen Instituts für technische Arbeitsschulung“ (DINTA) (1925) und deren Leiter bis zu ihrer NS-organisatorischen „Gleichschaltung“ 1935 in die „Deutsche Arbeitsfront“, wie folgt:

„War die Zweckbestimmung betrieblicher Arbeit bisher der private Gewinn im volkswirtschaftlichen Sinne, so ist heute jegliche Arbeit vom Gemeinnutzen her zu sehen. Die Unternehmerinitiative ist damit keineswegs ausgeschaltet, sondern lediglich mit höherem Ziel sittlich begründet und in ihrer Auswirkung bestärkt." “106

Die zynische Losung der NS-Sozialpolitik hieß folglich: keine Lohnerhöhung, dafür autosuggestive „Kraft durch Freude“ bei der Arbeit.

Welche wissenshistorische Kontinuität lässt sich zu de Mans Forschung über „Arbeitsfreude“ und seinem arbeitspsychologischen Konzept einer neuen Arbeitsethik, die er als eine „sittliche“ Arbeitspflicht aus „Schuld“ an der „Gemeinschaft“ definiert, zeigen? ${ }^{107}$ Die pejorative und elitäre Wortwahl des Sozialisten, die seinen Denkstil kennzeichnet, erstaunt in Bezug auf sein Forschungsthema, die „Arbeitsfreude“ in „der Arbeiterschaft“. „[D]ie relative Bedürfnislosigkeit der Masse" hätte ihn überrascht, schreibt der Sozialpsychologe blasiert. ${ }^{108} \mathrm{Zu}$ dieser Aussage ließ sich de Man am Ende seiner Ausführungen hinreißen, weil er die „Möglichkeit zum größten Glück“ im „Glück“ für andere „zu wirken“ erkannt habe. ${ }^{109}$ Von einem vermeintlich gesellschaftlich höher gestellten Standpunkt eines Wissenschaftlers aus formuliert de Man seine Ansichten in einem auffallend polemisch überspitzten Sprachstil. Anhand seiner Analysen zum „Herdentrieb“ und zum „Unterordnungsbedürfnis“ zeigt sich sein moralisierendes Werturteil, das ihn zu subjektiven Aussagen verleitet. Dabei changiert der sozialistisch eingestellte Psychologe zwischen Sympathiebekundung und Ab-

105 Ley 1936a, 48.

106 Arnhold 1936, 15.

107 Vgl. Man 1927, 183.

108 Vgl. ebd., 288.

109 Vgl. ebd., 289. 
grenzung gegenüber der Arbeiterschaft. Seine Ambivalenz wird an folgendem rhetorisch-provokativem Frage-Gegenfrage-Spiel deutlich:

„Oder gehen die ,niederen‘ Sorgen der Millionen, für die jeder Kampf ums Glück zunächst ein Kampf ums Brot ist, den ,geistigen` Menschen nichts an, weil er höheres Glück kennt? Wer darf so sprechen, solange er dieses Glück der Arbeit eben diesen Millionen verdankt, ohne die er selber nur ein nacktes, frierendes, hungriges, von den gemeinsten materiellen Sorgen besessenes Menschentier wäre?"110

Aus einer wissenschaftshistorischen Perspektive stellt sich die Frage, ob de Man mit seiner ambivalenten Haltung einen Einzelfall unter den Arbeitspsycho$\log ^{\star}$ innen darstellte. Inwiefern konnte sein Gedankengut aus bildungsbürgerlicher und sozialistischer Gesinnung, die im Rückblick teilweise bedenkliche Nähe zur NS-Arbeitsideologie Robert Leys und Carl Arnholds aufwies, die arbeitswissenschaftliche Forschung zur „Arbeitsfreude“ beeinflusst haben?

Wenn man der Kritik seines marxistischen Berufskollegen Siegfried Bernfeld (1892-1953), Psychoanalytiker, Erziehungswissenschaftler und Mitbegründer der Jugendpsychologie, Glauben schenken möchte, gab es eine große Anzahl sozialistisch-bürgerlich eingestellter Arbeitspsycholog ${ }^{\star}$ innen. ${ }^{111}$ Bernfeld machte zwei Strömungen innerhalb der Forschungsdisziplin aus: Die einen, welche Psychologie und Marxismus bzw. Sozialismus für ideologisch unvereinbar hielten und die anderen, „z. B. H. de Man“"112, welche vorgaben, einen sozialen Brückenschlag anzustreben. Was meinte Bernfeld damit? Die Psychologie werde mehrheitlich vom „ideologischen Standpunkt des Bürgertums“ aus betrieben. ${ }^{113}$ Folglich würde „bewußt oder unbewußt, mit oder gegen den Willen der einzelnen Forscher, im Sinne der Kapitalinteressen“ geforscht. ${ }^{114}$ Darin erkannte der Marxist das ideologische Dilemma der Arbeitspsychologie: Lange Zeit sei die „Arbeiterbewegung“ gegenüber der Arbeitspsychologie im Sinne Bernfelds marxistischem Klassenkampfdenken aus ideologischen Gründen misstrauisch gewesen. Als „neue Waffe“ gegen die „kapitalistischen Interesse[n]“ müsste sich die Arbeiterschaft jedoch in ihrer sozialistischen Bildung und im Betriebsalltag mit der Arbeitspsychologie auseinandersetzen. ${ }^{115}$ Zudem sollten Sozialist*innen arbeitspsychologische For-

110 Ebd., 190.

111 Zu Bernfelds Leben und Forschung vgl. u. a. Grasmeier 2019, Bernfeld 2018, Dudek 2012, Bernfeld 2010.

112 Bernfeld 1974, 142.

113 Vgl. ebd., 150.

114 Vgl. ebd., 150.

115 Vgl. ebd., 152. 
schung betreiben, um gemeinsam eine sozialpolitische Veränderung herbeiführen zu können:

„[...] die Psychologie muß von Sozialisten studiert und beherrscht, ihre Forschung muß von ihnen kontrolliert, ihre Anwendung muß aufmerksam verfolgt und auf ihre Funktion im Klassenkampf geprüft werden.“116

Tatsächlich beobachtete Bernfeld, wie die Psychologie im Verlauf der 1920er Jahre immer mehr ins „öffentliche Leben“ rückte und nicht länger als Elfenbeindisziplin ausschließlich „Fachleuten und Studenten“ vorbehalten blieb. ${ }^{117}$ Das Interesse der Arbeiterschaft, besonders im Kreis der Jugend, wachse stetig an, so der Jugendforscher. ${ }^{118}$ Die engagierte Berichterstattung über de Mans arbeitspsychologische Studie wäre ein Indiz für das große Interesse der jungen Hörer^innen an einem „Kampf um die Arbeitsfreude“.

Das von Bernfeld diskutierte ideologische Dilemma der Arbeiterpsychologie wird bei de Man offensichtlich: Der elitäre Wortlaut, den de Man in seiner Analyse wählte, wenn er den von Bernfeld zitierten Brückenschlag in seiner Studie zur „Arbeitsfreude“ beschrieb, offenbart sein ambivalentes Gedankengut: Es gäbe noch kein „sozialpädagogisch[es]“ Mittel, um eine „psychologische Brücke“ zwischen einer „höheren, rational begründeten Erwähnung einer kleinen geistigen Elite“, wozu der Autor sich zählte, und dem „dumpfen Gefühl der Masse für durch Gewohnheit geheilte Pflicht“" zu schlagen. ${ }^{119}$

Mit diesem Argument hat de Man die zukünftige Schaffung einer moralisierenden Arbeitsethik auf der Grundlage arbeitspsychologischer Erkenntnisse vor Augen. Diese sei nur dann erfolgreich, wenn sie anstelle intellektueller Argumente auf einer emotionalen Ebene die Masse anspreche. Den Grund dafür liefert de Man in einer biologistischen Argumentation, die seine herablassende Haltung gegenüber der Arbeiterschaft und ihrer angeblich massenpsychologisch „ins Blut übergangene[n], im Affekt verwurzelte[n] triebhafte[n] Gewohnheit“ aus einer gefühlshistorischen Perspektive einmal mehr offenbart: ${ }^{120}$

„Es geht nicht darum, neue Arbeitsfreuden zu erfinden; es genügt, die alte Arbeitsfreude aus ihren jetzigen Fesseln zu befreien und den sozialen Zielen einer neuen Führerschaft dienstbar zu machen." ${ }^{2121}$

116 Ebd., 150.

117 Ebd., 142.

118 Vgl. Ebd.

119 Vgl. Man 1927, 181.

120 Vgl. Ebd.

121 Ebd. 
Welche Aufgabe sollte nach de Man bei dieser emotionspolitischen Rhetorik die „Arbeiterfreude“ in der neuen Arbeitsethik übernehmen? Was stellte sich de Man unter den „sozialen Zielen“, die in den Dienst „einer neuen Führerschaft“ gestellt werden sollten, vor?

\begin{abstract}
„Um es gerade aus zu sagen: das Problem der Arbeitsfreude ist unlösbar, wenn man die sittliche Pflicht zur Arbeit für das Gemeinwohl nicht jedem anderen Arbeitsmotiv voranstellt. Ohne dieses Motiv die Arbeit zu einer reinen Freude gestalten zu wollen wäre eine Quadratur des Zirkels.“122
\end{abstract}

Im Eintrag zur „Arbeitspsychologie“, den de Man in Gieses Handwörterbuch der Arbeitswissenschaft 1930 publizierte, wird der Psychologe etwas konkreter: Der Arbeitspsychologe glaubt eine „sozialethische Inferiorisierung des Arbeiters“ erkannt zu haben. ${ }^{123}$ Dieses Inferioritätsgefühl des Arbeitertypus, wobei de Man einen verallgemeinerten Typus des „Industriearbeiters“ konstruierte, empfände sein „Arbeiterschicksal“ für weniger erstrebenswert als das „der besitzenden Klassen, das als das glücklichere gilt"“. ${ }^{124}$ An dieses Psychowissen über eine ,glücklichere“ Gesellschaftsschicht knüpft der Arbeitswissenschaftler sein rassistisches Gedankengut. De Man stellt zwischen „den Negern“ und deren angeblichem „Rasseninferioritätsgefühl“ einen Vergleich mit dem „Mohr Proletarier" an:

„Solange und sofern all dies der Fall ist [das Gefühl einer ,sozial inferiorisierten Klasse، (S. 284) zugehörig zu sein] könnte man ebensogut versuchen, den Negern ihr Rasseninferioritätsgefühl zu nehmen, indem man sie mit Traktaten zu überzeugen sucht, daß sie eine helle Haut haben. Dem Mohr Proletarier jedenfalls hält die soziale Wirklichkeit Spiegel genug vor, worin er seine Farbe erkennen kann.“125

Spätestens mit diesem Zitat wird eines deutlich: De Mans Studie als eine wissenshistorische Quelle über Glückswissen und einer Politik von ,Glückskulturen“ gelesen, ist mit Blick auf die Ambivalenz- und Kontinuitätsthese der vorliegenden Untersuchung erkenntnisreich. Denn in der bereits zitierten Rede von Robert Ley argumentiert dieser frappierend ähnlich, wenn er sagt:

„Der Mensch muß die Arbeit beherrschen. Minderwertigkeitskomplexe, die aus dem stolzen deutschen Arbeiter den knechtischen Proletarier machten, kommen auch zum Teil aus der Unzulänglichkeit des Menschen in seiner Stellung gegenüber der Arbeit. [...] Gerade der

122 Ebd., 183.

123 Vgl. Man 1930, 205.

124 Vgl. ebd. In der Studie zur „Arbeitsfreude“ vgl. u.a. Man 1927, $284 \mathrm{f}$.

125 Man 1927, $284 \mathrm{f}$. 
deutsche Mensch hat ein unerhört wertvolles Gut in seiner schöpferischen Art und seiner handwerklichen Begabung, und es muß erreicht werden, daß der Begriff , ungelernter Arbeiter tatsächlich nur den geringen Teil wirklich unintelligenter Menschen in Deutschland umfaßt.“126

Ley verbindet das arbeitspsychologische Wissen über einen empirisch angeblich nachweisbaren „Minderwertigkeitskomplex“ mit seiner emotionspolitischen Propagandastrategie über den vermeintlichen „Durchbruch der sozialen Ehre“ beim „deutschen Arbeiter“. Dieser „Durchbruch“ manifestierte sich laut NS-Propaganda in der Einführung des rassistischen „Gesetz[es] zur Ordnung der nationalen Arbeit“ am 12. Januar 1934, das sich explizit in Abkehr vom Marxismus und dem „liberalistische[n] Staat“ vollziehe. Diese Gesellschaftssysteme „glaubten, die Gemeinschaft entbehren und das Individuum allein in den Mittelpunkt des Geschehens stellen zu können. “127 Das übergeordnete Ziel von Leys emotionspolitischer NS-Arbeitsmoral sei, ,in dem Werk ,Kraft durch Freude‘ die Menschen dafür zu formen. “128 Wofür? Für „das Glück des Volkes“ im Sinne der NS-Ideologie (dem „Geist“ und der „Auffassung von der Welt“), aber nicht im Sinne des Individuums:

„Nicht die Gesetze, nicht die toten Buchstaben machen das Glück des Volkes, sondern allein der Geist und die Auffassung von der Welt, die der Mensch hat, formt sein Glück und formt seinen Wohlstand und gibt ihm den Segen."129

Zur glücksideologischen Formung der Menschen im Betrieb diene Ley angeblich das rassistische Arbeitsgesetz der „Gleichschaltung“, das dem Nationalsozialismus als repressives „Instrument“ diene, um den „anständigen Zeitgenossen mit unerbitterlicher Schärfe zu treffen und dem anständigen Menschen die Möglichkeit zur Gemeinschaft und zum Leben zu geben und damit den nationalsozialistischen Grundsätzen ,Gemeinschutz geht vor Eigennutz' und ,Dem Verdienst die Krone‘ zum Durchbruch zu verhelfen." ${ }^{\text {“130 }}$ Der Nationalsozialismus wurde von Ley dabei rhetorisch mit den „Menschen“ im Sinne der NS-Ideologie als ein totalitäres Herrschaftssystem gleichgesetzt. An diesem Beispiel zeigt sich, wie ein individuelles Glückstreben in der totalitären NS-Arbeitsmoral einem kollektivistischen Gemeinschaftsglück ideologisch zum Opfer fiel, um den Menschen, mit

126 Ley 1936a, 50.

127 Vgl. Ley 1940a, 62.

128 Vgl. ebd., 68.

129 Ebd., 68.

130 Vgl. ebd. 
den Worten de Mans, die „Dornenkrone“ eines „schmerzlich[en] Glück[s]“ aufzusetzen. ${ }^{131}$

In welcher wissenshistorischen Nähe Leys Argumentation zum arbeitspsychologischen Wissen über „Arbeitsfreude“ bei de Man lag, zeigt sich auch in Leys propagandistischer Rede über die Verschönerung des Arbeitsplatzes, wozu ihm insbesondere das neu geschaffene Amt „Schönheit der Arbeit“ dienen sollte. Abermals argumentierte Ley mit dem Schlagwort des „Minderwertigkeitsgefühl[s] des deutschen Arbeiters“, welches dem Eindruck einer „,dreckigen Fabrik““ geschuldet sei. ${ }^{132}$ Der Arbeitertypus würde wieder ein „Gefühl der Freude“133 an der Arbeit erlangen, wenn er stolz auf seine Arbeit sein könnte. Wie sollte diese „Arbeitsfreude“ laut der NS-Arbeitspsychologie geweckt werden? Das „Minderwertigkeitsgefühl“ würde sich relativieren, sobald der Arbeitertypus den „Wert“ seiner Tätigkeit in der gesellschaftlichen Anerkennung widergespiegelt sähe. ${ }^{134}$

Carl Arnhold, Professor an der Technischen Hochschule in Dresden, versuchte, Leys Arbeitspsychologie wissenschaftlich zu untermauern, indem er die Arbeitspsychologie in den Dienst des Nationalsozialismus stellte. Dies tat der Forscher im Wissen, damit den Anspruch Münsterbergs auf wissenschaftliche Objektivität und politische Neutralität einzubüßen:

„Nichts zeichnet die Zeitwende, die wir heute durchleben, eindringlicher als die Bemühung, die der Nationalsozialismus der Wiedererweckung eines deutschen Wertbewußtseins, eines deutschen Arbeitsstolzes, widmet. Man denke nur an den Aufbruch der schaffenden Menschen am 1. Mai, wo jeder persönlich erlebt, daß er wieder um seiner selbst willen, um seines Berufes und seiner Arbeit willen geehrt und geachtet wird. Der Bergmann, der Scholler, der Weber, sie alle sollen an diesem Tage fühlen und erleben, daß sie als deutsche Menschen und Werker die Achtung genießen, die ihnen als Kämpfer im Schützengraben der Arbeit zusteht. “135

Die Analogie zwischen Arbeitertypus und Soldatentypus treibt Arnhold propagandistisch auf die Spitze, wenn er in seinen arbeitswissenschaftlichen [!] „Erkenntnissen“ „rassenhygienisch“ argumentiert, worin das „Wertbewußtsein, eines deutschen Arbeitsstolzes“ begründet läge:

„Die Fundamente, auf denen wir all diese Arbeit aufbauen, bilden die Erkenntnisse, daß der deutsche Mensch, geworden aus seinen Vorfahren und geformt durch die Geschichte seiner

131 Vgl. Man 1927, 190.

132 Vgl. Ley 1935, 42.

133 Ebd.

134 Vgl. ebd. Zu „Minderwertigkeitsgefühl und Geltungsstreben“ in der Individualpsychologie um 1927 vgl. exemplarisch Adler 1928c.

135 Arnhold 1936, 33. 
Rasse, drei immer wieder hervortretende Grundwerte in seinem Blute hat: in seinem Fühlen, Denken und Handeln ist er Soldat, Handwerker und fantastischer Mensch.“136

Neben dem scheinbar wiedergewonnenen „Arbeitsstolz“ auf der Grundlage der totalitären „NS-Rassenideologie“, welche „Fühlen, Denken und Handeln“ des „gleichgeschalteten“ Menschen im Betrieb, der „Volksgemeinschaft“ „im kleinen“ ${ }^{137}$, verkörpere, legen Arnhold und Ley in wissenshistorischer Kontinuität zu de Man größten Wert auf die Arbeitsplatzverschönerung als einem Instrument der raumästhetischen NS-Gefühlspolitik eines Arbeitsglücks. Denn der Betrieb, so argumentiert Arnhold, sei kein „technisch-rationales Gebilde mehr, sondern eine lebendige, stark symbolhaltige, geistespolitisch geprägte Einheit, die ihren letzten Sinn im Staat hat.“"138 Die „Schönheit der Arbeit“, ob als Tätigkeit oder als synästhetisches Raumerlebnis im Kollektiv, sei ein weiterer emotionspolitischer Faktor, der die Industriebetriebe und das NS-Regime dem gemeinsamen Ziel, der gesteigerten Leistungsfähigkeit des Arbeitertypus durch gesteigerte „Arbeitsfreude“ (ohne Lohnerhöhung) näher bringen könne. Diese emotionspolitische „Betriebsgemeinschaft“"139 der gesteigerten „Arbeitsfreude“ sei die „Krönung“"140 der nationalsozialistischen Arbeitsideologie, so Arnhold in unverkennbarer wissenshistorischer Kontinuität zu de Man. Um die praktische Anwendbarkeit dieses arbeitspsychologischen Glückswissens zu demonstrieren, widmete der Bildband „Unter dem Sonnenrad. Ein Buch von Kraft durch Freude“, welcher 1938 von der „KdF“-Reichsamtsleitung herausgegeben wurde, der propagandistischen Demonstration der „Schönheit der Arbeit“ im „Dritten Reich“ ein zwanzigseitiges Kapitel inklusive Fotografien. ${ }^{141}$

An dieser Stelle der Argumentation angelangt, ist es entscheidend, der affirmativen NS-Gefühlspolitik mit ihren scheinbar ,neuen“ Erkenntnissen aus der NS-Arbeitswissenschaft den Spiegel der kritischen Geschichtswissenschaft vor Augen zu halten: Die „Schönheit der Arbeit“ im NS-Regime brachte nach 1933 mitunter die Zerschlagung und „arisierte“ „Gleichschaltung“ der Gewerkschaften, das Verbot der Betriebsräte sowie die Wiedereinführung alter patriarchaler Betriebssysteme mit sich. Die produktions- und leistungssteigernde Wirtschaftlichkeit der Industriebetriebe fand mit Hilfe psychotechnischen Wissens auf Kosten

136 Ebd., 28.

137 „Damit wird der Betrieb das Abbild und Sinnbild des nationalsozialistischen Staats im kleinen.“ Ebd., 11.

138 Vgl. Arnhold 1936, 11.

139 Ebd., 57.

140 Ebd.

141 Vgl. Busch 1938, „Schönheit der Arbeit“, 59-79. 
der Belegschaft statt. ${ }^{142}$ Letzteres hatten NS-Propagandisten wie Ley den Betrieben und Arbeitswissenschaftlern der Weimarer Republik vorgeworfen. Im Sinne der rassistischen Arbeitsideologie hatten die NS-Funktionäre jedoch gemeinsam mit den Unternehmern und den NS-Arbeitspsycholog*innen die sozialpolitischen Arbeitsbedingungen in den Betrieben auf eine Weise radikalisiert, wie es in keinem Verhältnis zur psychotechnischen Forschungstätigkeit und Betriebspolitik in der Weimarer Republik steht. Trotz eindeutiger ideologischer Brüche, die zu einer „rassenhygienischen“ Radikalisierung und moralischen Entgrenzung der wissenschaftlichen Tätigkeit führten, sind nach 1933 in der arbeitswissenschaftlichen Forschung gewisse wissenshistorische Kontinuitäten von arbeitspsychologischem Glückswissen nachzuweisen, wie exemplarisch anhand de Mans Studie zur „Arbeitsfreude“ aufgezeigt wurde.

Die arbeitspsychologische Studie de Mans bildet folglich ein emotionspolitisches Wissensspektrum von den individuellen, emanzipatorischen (und nicht ,bedürfnislosen' oder unterwerfungswilligen) Forderungen der befragten Arbeiterschaft bis $\mathrm{zu}$ den angeblich objektiven Schlussfolgerungen des ambivalent rassistisch-bürgerlich-sozialistisch argumentierenden Arbeitspsychologen über einen „neuen Arbeitsethos“ als „sittlicher Pflicht“ bzw. aufgrund „soziale[n] Pflichtgefühl[s]“ “ im Dienst einer Gemeinschaft ab. ${ }^{143}$ Der Vergleich zu Leys Propagandareden und Arnholds arbeitswissenschaftlichen Schriften über die Bedeutung der „Arbeitsfreude“ macht deutlich, welche ambivalente Wissenskontinuität de Mans Studien als ein „zusammenhängende[s] Geflecht“144 von „Tatsachen“145 in Beziehung zu den „Denkkollektiven“146 im Kontext der affirmativen NS-Gefühlspolitik erfuhr. ${ }^{147}$ De Mans „Denkstil“ war selbst ambivalent. Aus einer biografischen Perspektive betrachtet, entspricht dies seiner ambivalenten Persönlichkeit, die sich in de Mans bewegter Lebensgeschichte widerspiegelt. In seiner Doppelrolle als Arbeitspsychologe und Sozialist vertrat er u.a. als Minister in den 1930er und 1940er Jahre repräsentative Ämter in der belgischen Regierung. 1940 riet er dem belgischen König Leopold II. beim Einmarsch der

$142 \mathrm{Zu}$ den NS-politischen und gesellschaftlichen Umstrukturierungen betreffend der arbeitswissenschaftlichen Forschung im Nationalsozialismus vgl. Raehlmann 2014, 125f. Auch Raehlmann sieht in den NS-arbeitswissenschaftlichen Arbeitsfeldern, die sie untersuchte, namentlich Ernährungsforschung, Gasschutz und Sport, auch Kontinuität zu bisherigen und späteren Forschungen.

143 Vgl. Man 1927, 183.

144 Vgl. Fleck 2008, 286.

145 Vgl. ebd.

146 Vgl. ebd.

147 Dieser Aspekt wird in den Kapiteln 12 und 16.3 weiterführend untersucht. 
deutschen Truppen zur Kooperation. Kurze Zeit darauf erhielt er ein Rede- und Publikationsverbot im „Dritten Reich“ und wurde nach dem Zweiten Weltkrieg aufgrund seiner Kollaboration mit dem NS-Regime von einem belgischen Gericht zu 20 Jahren Haft verurteilt. ${ }^{148}$

Vor diesem Hintergrund gewinnt de Mans Studie erneut an wissens- und emotionspolitischer Brisanz: De Mans massenpsychologische Beobachtungen prognostizieren im Kapitel zum „soziale[n] Pflichtgefühl“ gesellschaftspolitische Entwicklungen, die sich um 1933 in der Weimarer Republik zum Teil bewahrheiten sollten. De Man diagnostiziert bei denjenigen, die über „Arbeitsunlust“ klagten, das fehlende Bewusstsein der eigenen Arbeitspflicht gegenüber der Gemeinschaft, was er auf die rationalisierte, rein gewinnorientierte Arbeitskultur und die überschätzte Bedeutung des marxistischen „Klassenbewußtseins“ in der Arbeiterschaft zurückführt. Spätestens seit dem Ersten Weltkrieg böten neben „kirchlichen Glaubensgemeinschaften“ vor allem „die nationalen Schicksalsgemeinschaften“ Alternativen an, um das Bedürfnis nach den zerstörten älteren „Gemeinschaftsverbindungen (Lokalpatriotismus, ländliche Nachbarschaft, organisierter Stand, Zunft usw.)“ zu kompensieren. ${ }^{149}$ Die Folge sei eine „außerordentliche Steigerung des Nationalgefühls bei den Maßen [sic]“150. Die „Suggestionskur der Moral- und Pflichtpredigt“151 verspreche, „den Massen die höhere Arbeitsfreude aus Pflichterfüllung“ gegenüber der Gemeinschaft zu ermöglichen. In der Weimarer Republik werde der „Gemeinschaftsbegriff“ im Sinne der „Nationalinteresse[n]“ über die „Propaganda für die freiwillige Steigerung der Arbeitsleistung“ gespannt. De Man sieht diese Entwicklung, die er namentlich bei den gewerkschaftlichen Ideologien beobachtet, durchaus kritisch. ${ }^{152}$ Das propagierte „Ziel der Hebung des Nationalwohlstands“ zeuge von den „viel zu starke[n] Leidenschaften der Kriegszeit“, die kurzweilig eine autoritäre „,Führerangelegenheit““ impliziere, statt eine „soziale Pflichterfüllung“ zu fordern, wie sie de Man in seiner Arbeitsethik vorschwebte. ${ }^{153}$ Diese Form der „Verwandlung der Massenmotive bei der Arbeit“ laufe Gefahr der Arbeiterschaft eine „Dornenkrone“ eines „schmerzlich[en] Glück[s]“ aufzusetzen. ${ }^{154}$ Was meint de Man damit? Wie schon Baerwald argumentiert auch de Man, dass nur der individuelle Selbstzweck

148 Zur Biografie s. früher im Kap. 10.4.

149 Vgl. Man 1927, 187.

150 Vgl. ebd.

151 Vgl. ebd., 190.

152 Der ideologiepolitische Wandel einiger Gewerkschaftsvertreter wird exemplarisch am Beispiel August Winnigs im Kapitel 11.2 aufgezeigt.

153 Vgl. Man 1927, $187 \mathrm{f}$.

154 Vgl. ebd., 190. 
es erlaubt, ein „schmerzliche[s] Glück“ zu erdulden. ${ }^{155}$ Abermals in beunruhigender Nähe zur NS-Rhetorik der freiwilligen „Opferbereitschaft“ zeigt sich de Mans Ambivalenz:

„Wie es eine Rangordnung der Freuden und Glückswerte gibt, so gibt es auch als massen-
psychologische Wirklichkeit eine Rangordnung der Menschen, die zum Genuß dieser Werte
die Veranlagerung und Kraft haben. [...] Wohl ist die Opferwollust, die aus Leiden Freude
macht, das höchste Glück, das einem Menschen beschieden sein kann. Aber dazu muß er
kräftig genug sein, und den Beweis dieser Kraft kann er immer nur selber erbringen, und
zwar durch die selbstgewünschte Tat. Das ist eine Forderung, die man immer nur an sich
selber stellen kann. Wer sie an andere stellen wollte, würde sich dem Verdacht aussetzen,
daß die Leiden des anderen ihn weniger schmerzen, als wenn er sie selbst empfände.“156

Wie lässt sich dieser politische Aspekt des arbeitspsychologischen Glückswissens über „Arbeitsfreude“ bei de Man vor dem Hintergrund der anwachsenden nationalsozialistischen Bewegung Ende der 1920er Jahre interpretieren? Der individuelle „Drang“ nach Arbeitsglück konnte Gefahr laufen, von einer Glückspropaganda überholt zu werden, die das „Glück“ der Einzelperson zu Gunsten eines „Glücks“ der nationalen „Schicksalsgemeinschaft“ opferte. ${ }^{157}$ Gewisse Aspekte dieser Interpretation spiegeln sich auch in de Mans späteren Schriften zum „Sozialismus und Nationalfacismus“ (1931) und „Massen und Führer“ (1932) wider. ${ }^{158}$ Angesichts des drastischen Zuwachs von nationalsozialistischen Wählerstimmen um 1930 beschäftigte sich der Sozialpsychologe intensiv mit der NS-Bewegung. ${ }^{159}$ In Anbetracht des gravierenden Bedeutungsverlusts der sozialistischen und sozialdemokratischen Parteien fragte de Man „vom Standorte des Intellektuellen“160 nach den Gründen für den politischen Erfolg der nationalsozialistischen Massenbewegung. Im Sinne seiner strittigen Triebtheorie, die er bereits 1927 für die „Arbeitsfreude“ entwickelt hatte, sah der Sozialpsychologe im „Drang zu Mythos und Utopie“, im „Trieb zur Aktivierung der Masse“ sowie im „Verlangen nach Führerpersönlichkeit“ mögliche Antworten. ${ }^{161}$ In der Schrift „Massen und Führer“, die er als Vortrag für die Tagung des „Internationalen Verbandes für kultu-

155 Vgl. Man 1927, $189 \mathrm{f}$.

156 Ebd.

157 Vgl. ebd., 187.

158 Vgl. Man 1931 und Man 1932b.

159 Die Schrift „Sozialismus und Facismus“ ging auf einen Vortrag zurück, den er bereits am 12.12.1930 „,vor der Ortsgruppe Berlin des Kulturbundes“ gehalten hatte. Vgl. Man 1931, „Vorbemerkung“, o.S.

160 Vgl. Man 1931, „Vorbemerkung“, o.S.

161 Vgl. Man 1931, 48. Zum Utopiediskurs und der Ideengeschichte in der Weimarer Republik vgl. Graf 2003, vgl. Nolte 2003. 
relle Zusammenarbeit“ im Oktober 1930 in Zürich verfasst und schließlich in Wien vor dem „Kulturbund“ gehalten hatte, versuchte er, die „Massenbildung und die Führerfunktion“ im Kontext der historischen Entwicklung von „Herrschaftsformen“ $\mathrm{zu}$ analysieren. ${ }^{162}$

De Man entwarf 1931 jedoch „zwei Enden“ der „Geschichte“. Die Errichtung einer NS-Diktatur hielt der Sozialpsychologe nach wie vor für abwendbar; „Wie es im Manuskript vieler zeitgenössischer Romanschriftsteller vorkommen soll, bevor sie sich für das happy oder unhappy end entschieden haben. " ${ }^{163}$ Im Unterschied zum fiktionalen Erzählen sei der Ausgang der realpolitischen Geschichte der Weimarer Republik selbstverständlich keine Frage des subjektiven, ästhetischen Geschmacks, sondern eine moralische:

„Die Kontrastierung vom happy end mit dem unhappy end hat nur den Sinn, klar zu machen, daß am Ende derartiger Betrachtungen immer eine Wahl zu stehen hat - eine Wahl nicht bloß der privaten Gesinnung, sondern ein Partei-Ergreifen, eine Entscheidung zum Handeln." ${ }^{164}$

Die moralische Frage des „Partei-Ergreifen[s]“, wie es die Wählerstimmenabgabe für eine bestimmte Partei darstelle, ist „eine Entscheidung zum Handeln“, die jede Einzelperson für sich beantworten müsse. Inwieweit de Man mit seinen janusköpfigen Zukunftsentwürfen recht haben sollte, beziehungsweise mit welchem Glückswissen die nationalsozialistische Arbeitspolitik die Erkenntnisse der arbeitswissenschaftlichen Forschungen für ihre Propagandazwecke nutzbar machen sollte, wird in den folgenden Kapiteln weiter aufgezeigt.

\section{5 „Ethos der Arbeit“: Zur „Arbeitsfreude“ im „Handwörterbuch der Arbeitswissenschaft"}

Um den rezeptionshistorischen Stellenwert der „Arbeitsfreude“ in der Psychotechnik wissenshistorisch überprüfen zu können, lohnt sich ein erneuerter Blick in das offizielle „Handwörterbuch der Arbeitswissenschaft“ von 1927. Wie wurde im selben Jahr, in dem de Mans Studien erschienen, „Arbeitsfreude“ lexikalisch

162 Vgl. Man 1932a, 3. In seinem Vorwort (Oktober 1932) schreibt de Man, dass die Zürcher Tagung zum Thema „Geist und Macht“ aus „wirtschaftlichen Gründen“ abgesagt wurde, sein Vortrag jedoch 1931 in der „Europäischen Revue“, November/Dezember-Heft erschienen sei.Vgl. Man 1932a, 4.

163 Vgl. Man 1931, 61.

164 Ebd. 
definiert? Welche Ähnlichkeiten und Unterschiede lassen sich zu dem bereits untersuchten Forschungsstand und den Ratgebermeinungen erkennen und welche Bedeutung spielten dabei gesellschaftspolitische Komponenten? Im Lexikoneintrag zu „Ethos der Arbeit“ grenzte der Neukantianer Georg Mehlis (18781942) zunächst einen „objektiven Wert des Arbeitsprodukts“ von einem „subjektiven Wert der Arbeitsfunktion oder Arbeitstätigkeit“ ab. ${ }^{165}$ Letzterer könne von „einem ethischen Wert der Arbeitspflicht“ als Pflichterfüllung und dem „hedonistischen Wert der Arbeitsfreude“ unterschieden werden. ${ }^{166}$ Dieser „Wert“ entfalte seine Wirkung als „Lustgefühl“ oder „als „Gefühl der Zufriedenheit und Befriedigung“ auf den Körper der Arbeiter*innen. ${ }^{167}$ Nach Mehlis besagt der „Ethos der Arbeit“, „daß mit ihr [der Arbeit] Glück und Segen für den arbeitenden Menschen zusammengeht“". 168

Gefühlshistorisch betrachtet war die Forderung nach „Freude“ und „Glück“, welche die tägliche Arbeit dem Individuum bescheren sollte, keine Erfindung der Psychotechniker`innen. Diese war beispielsweise im marxistischen und utilitaristischen Diskurs der Arbeiterbewegung des 19. Jahrhunderts bereits thematisiert worden. Was den Gefühlsdiskurs der Psychotechnik in den 1920er Jahre auszeichnete, war die Verknüpfung sozialpolitischer Anliegen mit Glückswissen aus empirischen Forschungsresultaten der Arbeitspsychologie im Kontext der sich im Aufbau befindenden, demokratischen Weimarer Republik. Problematisch war die Verbindung von gesellschaftspolitischen Zukunftsvisionen zu einer „Glück“ verheißenden Arbeitsgesellschaft mit dem Objektivitätsanspruch empirischer Wissenschaftlichkeit.

Aufhorchen lässt in Mehlis Eintrag zum „Ethos der Arbeit“ folgender Passus, der die ideologische Nähe seines Konzepts zur NS-Ideologie im Sinne Robert Leys („,Kraft durch Freude“ ist das Reglement, nach dem exerziert wird“169), erahnen lässt: „Wenn es auch Arbeit gibt, die schädlich für Seele und Körper ist, so hat sie doch im allgemeinen diese heilsame Funktion, daß sie das beste Mittel ist, um den Menschen von jener Leere und Monotonie zu befreien [...]“, versichert Mehlis den Leser*innen. ${ }^{170}$ Wie argumentiert Mehlis? Indem er auf das Glückswissen eines moralphilosophischen Arbeitsethos zurückgreift:

165 Vgl. Mehlis 1927, 1703.

166 Vgl. ebd.

167 Vgl. ebd.

168 Vgl. ebd., 1704.

169 Ley 1939, 269.

170 Vgl. Mehlis 1927, 1703. 
„Der Mensch, der arbeitet, weiß, daß er zu etwas da ist, daß er eine Aufgabe zu lösen hat. Er kann Sinn mit seinem Leben verbinden, indem er sein Tun als zweckvoll im Dienst der Gemeinschaft betrachtet. Das Glück der Berufstätigkeit liegt in dem Bewußtsein des sinnvollen Tuns. E. [Der Ethos] der Arbeit besagt, daß mit ihr Glück und Segen für den arbeitenden Menschen zusammengeht.“"171

Mag die Arbeit noch so entbehrlich für die Psyche und Physis der Arbeiter*innen sein. Heilsames „Glück“ beschere sie den Ausführenden allemal, wenn sie „im Dienst der Gemeinschaft“ vollzogen würde. Damit erfülle die Arbeit einen höheren, sozialpolitischen Zweck, welcher den Schaden „für Seele und Körper“ legitimieren könne, so die moralisch fragwürdige Argumentation in Mehlis’ Arbeitsethik.

Die wissenshistorische Kontinuität zum Gedankengut einer glücksideologischen Arbeitsethik lautete im arbeitspsychologischen Dienst einer „arisierten“ Gemeinschaft in Leys Rede wie folgt:

„Die Partei ist die Hüterin unserer Welt, die Partei ist die verschworene Gemeinschaft einer Auslese von Menschen, einer Minderheit, von Tapferen und Mutigen und Einsatzbereiten und Opferbereiten. Die Partei wird die Menschen, die in ihr sind, hart anfassen. Dafür gibt diese Partei auch den Menschen das hohe Glück, an diesem Bau Deutschland bauen zu dürfen. Die Partei gibt das höchste Glück, sie verlangt aber auch die größten Opfer!

Die Arbeitsfront ist die Gemeinde. In ihr wird die Weltanschauung zur Anwendung gebracht. Sie gleicht die Interessen aus, sie richtet vor allen Dingen die Gemeinschaft auf und treibt diese Gemeinschaft zur höchsten Leistung.“172

In Kapitel „Falsche Ideale“ vertrat der bereits zitierte Arbeitspsychologe und Ratgeberautor Baerwald (Kap.10.1) eine entgegengesetzte Auffassung und betonte dabei die „Freude am Schaffen als Selbstzweck“:

„[Dem Volksgenossen (der heutigen Gesellschaft) soll] ein Kollektivleben eingeräumt werden, weil man ihn um den Kern seines individuellen Lebens gebracht hat. [...] Restlos wird man aber mit einem solchen Ersatz des Einzelich [sic] durch das Gesamtich nie auskommen.“173

Im Unterschied zum Glücksratgeberautor Baerwald und dem Arbeitspsychologen de Man, die selbst bei mühsamen Arbeitstätigkeiten ausschließlich den Selbstzweck der „Arbeitsfreude“ für das Individuum idealisierend betonen, sah Mehlis -

171 Ebd., 1704.

172 Ley 1939, $257 \mathrm{f}$.

173 Baerwald 1933, 222. 
ähnlich wie Ley - das „Glück der Berufstätigkeit“ im Wissen um dessen Zweckmäßigkeit für die „Gemeinschaft“:

„[...] Wo aber die Ausführung der Arbeit selber das Gefühl von Freude und Glück nicht aufkommen läßt, weil die Schwere der Arbeit als gar zu große Mühe oder gar als Qual empfunden wird, wird die vollendete Arbeit umsomehr mit Lustgefühlen verbunden sein. [...] Er [,der Arbeiter'] kann Sinn mit seinem Leben verbinden, indem er sein Tun als zweckvoll im Dienst der Gemeinschaft betrachtet. Das Glück der Berufstätigkeit liegt in dem Bewußtsein des sinnvollen Tuns. “174

Welche sozialpolitische Einstellung hatte dieser Autor und warum entschied sich die Herausgeberschaft des offiziellen „Handwörterbuchs für Arbeitswissenschaft“ gerade für ihn? Mehlis lebte 1927, als das Handbuch erschien, in Italien und war mit der Niederschrift seiner Werke „Die Idee Mussolinis und der Sinn des Faschismus“ (1928) und „Der Staat Mussolinis. Die Verwirklichung des kollektiven Gemeinschaftsgedankens“ (1929) beschäftigt. ${ }^{175}$ Der neukantianische Philosoph, der zeitweilig an der Universität Freiburg i. B. gelehrt hatte, zeigte sich vom italienischen Faschismus und dessen für die Arbeiterschaft 1925 neu geschaffene nationale Freizeit- und Erholungsorganisation „Nach der Arbeit“ („Opera Nazionale Dopolabora“) beeindruckt. Er war begeistert, als nach 1933 der nationalsozialistische „Führerstaat“ für Deutschland Realität wurde, wovon seine Schriften „Freiheit und Faschismus“ (1934) und später „Führer und Volksgemeinschaft“ (1941) zeugen. ${ }^{176}$

1927 definiert Mehlis das „Glück“ des „arbeitenden Menschen“ nicht aus der Sicht des Individuums, sondern im Sinne eines totalitären „Glücks“ für das „Kollektivwesen Volk und Staat“: „E. [Ethos] der Arbeit besagt, daß mit ihr Glück und Segen für den arbeitenden Menschen zusammengeht. [...] Was für den einzelnen [sic] gilt, gilt für jede Gemeinschaftsform und so auch besonders für die Kollektivwesen von Volk und Staat. “177 Diese totalitäre Vereinnahmung eines Arbeitsglücks war im Sinne des Herausgebers Fritz Giese, der die Psychotechnik nach 1933 in den Dienst der angewandten NS-Arbeitspsychologie und der nationalsozialistischen „Rassenhygiene“ in den „arisierten“ Betrieben stellen sollte, wie in den Kapiteln 11-14 aufgezeigt wird.

174 Mehlis 1927, 1704.

175 Vgl. Mehlis und Mussolini 1928, vgl. Mehlis 1929.

176 Vgl. Mehlis 1934, vgl. Mehlis 1941.

177 Vgl. Mehlis 1927, 1704. 


\subsection{Der Streit um eine arbeitspsychologische Sozialpolitik der „Arbeitsfreude“}

Die Definitionsbeispiele einer Arbeitsmoral und einer „Arbeitsfreude“ aus dem „Handwörterbuch der Arbeitswissenschaft“ von 1927 sollten nun nicht den Eindruck erwecken, dass die Psychotechnik zwangsläufig als nationalsozialistische Arbeitspolitik im Kontext der NS-,,Rassenhygiene“ ihre Anwendung finden musste. ${ }^{178}$ Das Gegenteil war der Fall.

Bereits Münsterberg warnte vor den gesellschaftspolitischen Folgen einer übereilten Anwendung der noch jungen und wenig erprobten Psychotechnik in der Betriebspraxis: „Das psychologische Wissen kann immer nur besagen, daß, wenn wir dieses Ziel wollen, wir diesen Weg einschlagen müssen. “" ${ }^{179}$ Die Prämisse eines wissenschaftlichen Objektivitätsanspruchs stellte Münsterberg seiner Wissenschaftstechnik folglich voraus. Eine soziale, rechtliche oder moralische Verantwortlichkeit für die Ausübung der Psychotechnik im Betrieb wies der Psychologe von sich. ${ }^{180}$ Lediglich die Wissenspraktiken, um die Ziele der Produktivitätssteigerung durch Rationalisierung zu erreichen, böten die Arbeitspsycholog^innen an, so Münsterbergs Argumentation. ${ }^{181}$ Daher müssten die Anwendungsziele sowie deren „praktische Bewertung“ im Voraus von moralischen Wertvorstellungen über den Arbeitertypus als menschliches Wesen und nicht als ausbeutbare Maschine geleitet sein. „Die Psychotechnik steht nicht im Dienste einer Partei, sondern ausschließlich im Dienste der Kultur“, behauptet Münsterberg. Angesichts der wenige Jahre später erstarkenden nationalsozialistischen Bewegung ist diese Schlussfolgerung im Nachhinein als kurzsichtig zu bewerten. ${ }^{182}$

Die Meinung, dass die Psychotechnik sehr wohl von moralischen und ethischen Vorstellungen getragen und nicht ausschließlich einem wissenschaftlichen

178 S. Kap. 11 und 12.

179 Münsterberg 1920, 39.

180 Zur Veranschaulichung seiner Argumentation zog er den Fall einer Geständnisherbeiführung vor Gericht heran: „Der Psychologe mag dem Richter zeigen, durch welche psychologischen Hilfsmittel beispielsweise ein Geständnis erwirkt werden mag; aber ob es recht oder unrecht ist, ob es gesetzlich zulässig oder gesetzlich unerlaubt ist, von dem Angeklagten ohne sein Wissen und Wollen zu erzwingen, das ist eine Frage, die den Psychologen nichts angeht, und zu deren Lösung [...] auch nichts beizutragen weiß. Er weiß nur, wie das Ziel erreicht werden kann; er hat kein Recht darüber zu urteilen, welchen Wert das Ziel besitzt. Das ist nicht etwa eine Schwäche der heutigen Psychologie [...] diese Verzichtserklärung ist sämtlichen Kausalwissenschaften gemeinsam.“ Münsterberg 1920, $39 \mathrm{f}$.

181 Vgl. Münsterberg 1920, 40.

182 Vgl. Münsterberg 1919, 88. 
Objektivitätsprinzip verpflichtet sei, vertrat hingegen der Psychiater Eliasberg vehement:

„Die Psychotechnik hat mit dem gesamten Wirtschaftsleben die eudämonistische Grundanschauung gemein. Sie will das Glück der Gesamtheit durch Produktivitätssteigerung erhöhen, sie will auch gleichzeitig das Glück des Produzenten steigern und zwar in der Berufstätigkeit, wie auch außerhalb derselben. Für die Psychotechnik ist der Produzent ein Mensch im vollen Sinne des Wortes, der, unbeschadet der Notwendigkeiten der Produktion, sein Anrecht auf Glück hat.“183

In Referenz auf ein in der Antike entwickeltes eudämonistisches Weltbild sah Eliasberg in dieser Passage im Erlangen von „Glück“ das höchste Lebensziel. „Glück“ sei nicht nur den machtpolitischen und vermögenden Eliten vorbehalten, sondern „Glück“ steigere sich sowohl in der Berufstätigkeit des „Produzenten“ und wirke sich darüber hinaus auf die außerberufliche Lebensqualität aus. Eliasbergs Glückswissen lag folglich die Vorstellung einer Gesellschaftsutopie zugrunde, die auf der Grundlage wissenschaftlicher Erkenntnisse einer angewandten Glückspsychologie mittels Psychotechnik herbeigeführt werden könnte. Dieses utilitaristische Gedankengut knüpft wissenshistorisch an die Überlegungen des Juristen und Moralphilosophen Jeremy Benthams (1748-1832) an, der in seiner Ethik über „Das größte Glück der größten Zahl“ das Interessenverhältnis zwischen Individuum und Gesellschaft problematisierte. ${ }^{184}$ Mit diesem utilitaristischen Argument unterschied sich der Arbeitswissenschaftler Eliasberg deutlich vom Psychologen und Ratgeberautor Baerwald. Letzterer bezeichnete gerade nicht „Eudämonie“, sondern das „Arbeitsglück“ als Selbstzweck im Glauben an „amor fati“ als das „höchste Glück“. ${ }^{185}$

Einen zentralen Kritikpunkt an seiner eigenen Methodik sah Eliasberg in der gesellschaftspolitischen und moralischen Verantwortung, in welcher Arbeitspsycholog^innen aufgrund der politischen Wirkungsmacht ihrer Psychotechniken auf die „Gesamtkultur“186 standen. Seine Überlegungen zu den „Richtungen u. Entwicklungstendenzen in der Arbeitswissenschaft. II“ schloss er mit einer selbstkritischen Problematisierung seiner eigenen empirischen Erhebungen zur betrieblichen Arbeitsmotivation:

183 Eliasberg 1926a, 80.

184, „[...] this fundamental axiom, it is the greatest happiness of the greatest number that is the measure of right and wrong, [...]. “ Bentham 1988, 3. Zur Problematik einer sozialen Umsetzung der Psychotechnik exemplarisch bei Münsterberg vgl. Münsterberg 1920, 192f., 194-198.

185 Vgl. Baerwald 1933, 218 f., 271.

186 Eliasberg 1926b, 732. 
„Der entscheidende Grund, warum das Problem der Heilpädagogik der Motivationsstufen eindeutig nicht gelöst werden kann, liegt in der Natur der Motivation der Arbeit überhaupt. Wir haben gesehen, daß in dem individuellen Erlebnis der Motivation individuelle, kollektive, institutionelle Momente sich unlösbar verschlingen und daß noch zu alledem der Reflex der Gesamtkultur tritt. Daß alles theoretisch auf einen Generalnenner zu bringen wollen, ist undenkbar. Darum wird das Problem einer Heilpädagogik immer ein politisches, ein Machtproblem bleiben. Der Sozialtherapeut wird nicht nur Sozialingenieur, sondern auch Politiker sein müssen." ${ }^{187}$

Eliasberg beleuchtet in dieser Passage die emotionspolitische Komplexität der Arbeitsmotivation, mit welcher sich die arbeitswissenschaftliche Psychotechnik und deren heilpädagogische Ansätze in der Praxis konfrontiert sahen. ,[I]ndividuelle, kollektive und institutionelle Momente" sowie außerbetriebliche Einflüsse aus der „Gesamtkultur“ würden auf das Motivationserlebnis der Arbeiter^innen einwirken, so Eliasberg. Anders als de Man, der aufgrund ähnlicher empirischer Resultate seine strittige „Triebtheorie“ über den „Drang zur Arbeitsfreude“ entwarf, verzichtete Eliasberg auf eine ausführliche Theoriebildung. Den Anspruch gesellschaftspolitischer Neutralität und wissenschaftlicher Objektivität an die Psychotechnik, den Münsterberg vertrat, und die damit verbundene Hoffnung, ein wissenschaftliches Vermittlungsinstrument zwischen den Interessen der Arbeitgeber- und -nehmer^innen gefunden zu haben, sah Eliasberg zwangsläufig in einem machtpolitischen Spannungsfeld. In dessen Mitte hatte sich der „Sozialingenieur" in seiner ,Rolle“ als Sozialpolitiker zu positionieren.

Wie gelangte Eliasberg zu dieser folgenschweren Erkenntnis? Eliasbergs Einschätzung beruhte unmittelbar auf seinen empirischen Beobachtungen: Die psychotechnischen Prüfstellen der Betriebe wurden oft nicht von unabhängigen Arbeitswissenschaftler`innen, sondern von psychologisch geschulten, betriebsinternen Berufsingenieur*innen ausgeübt. ${ }^{188}$ Münsterbergs Forderung nach wissenschaftlicher Objektivität, „politischer“ Neutralität sowie moralischer Zielsetzung verfingen sich in der Realität in Widersprüchen, namentlich den von Eliasberg erkannten politischen Machtkonstellationen innerhalb eines Betriebs. Eignungsprüfer`innen konnten sich gegenüber der Forderung nach betrieblicher

187 Ebd. [Die Kursivsetzung wurde von der Verfasserin nachträglich ergänzt].

188 Auch die Schweizer Arbeitswissenschaftlerin Franziska Baumgarten kritisierte das betriebsinterne Verfahren und stellte dabei psychologische Aspekte in den Vordergrund: „Hervorgehoben werden muss [...] daß die Forderung der [Eignungsprüfung] hauptsächlich von Ingenieuren oder denjenigen Psychologen stammt, die fast ganz zur betriebswirtschaftlichen Arbeit übergegangen sind [S. Fußnote 1: „Auf Motive der Profitmacherei will ich gar nicht eingehen.“]. Sie sehen die Gesellschaft als einen geschlossenen Betrieb an und vergessen, daß ein Betrieb eine ganz andere psychische und wirtschaftliche Struktur darstellt.“ Vgl. Baumgarten 1931, $16 \mathrm{f.}$ 
Effizienz- und Produktionssteigerung kaum neutral verhalten. Aus Sicht der Unternehmensführung war dies zuweilen auch gar nicht erwünscht. So zeigt Ursula Nienhaus beispielsweise, wie die Psychotechnik im Auftrag des Telegraphendirektors O. Oliver der Deutschen Post in Berlin-Wilmersdorf zum Zweck der Rationalisierung von Arbeitsprozessen, zur finanziellen Einsparung und zum sukzessiven Stellenabbau auf die Frauenarbeit angewandt wurde. Ziel war es, den Telefonist*innen einen automatisierten Arbeitsprozess anzutrainieren, sodass sie auch bei erhöhter Leistungsabfrage keine Ermüdungs- oder Erschöpfungserscheinungen aufweisen würden. Bei der psychotechnischen Eignungsprüfung hatten neben physischen auch charakterliche Eigenschaften eine Bedeutung, welche die Leistungsfähigkeit der Telefonistinnen angeblich beeinflussen würden. ${ }^{189}$

\subsection{Psychotechnik im Betrieb: Der Traum vom rationalisierten „Arbeitsglück“}

Ungehindert dieser Vielzahl an Paradoxien, die symptomatisch für die unter der Rationalisierungsbewegung ab Mitte der 1920er Jahre zu verzeichnenden Zunah$m e$ an Erschöpfungserscheinungen in der Arbeiterschaft stehen dürften, genoss die Psychotechnik anfänglich großen gesellschaftlichen Zuspruch. Wie lässt sich dieses Phänomen wissenshistorisch erklären? Im Gegensatz zur autoritären Gesellschaftsordnung des Wilhelminischen Kaiserreichs mit ihren aristokratischen und militärischen Karriereprivilegien sollte dank der Psychotechnik eine neue, demokratische Ära sozialen „Glücks“ in der Weimarer Republik eingeläutet werden. Dieses spezifische Glückswissen fand bis Mitte der 1920er Jahre im politischen Spektrum von Links bis Rechts, bei Industriellen, Arbeiter*innen, Gewerkschafter^innen, Politiker^innen und der Regierung Anklang. Große Erwartungen wurden in die wissenschaftliche Dienstleistung der Psychotechnik gelegt, die nicht nur wissenschaftlich institutionalisiert, sondern auch staatlich finanziert und von der Regierungsverwaltung in Anspruch genommen wurde. ${ }^{190}$

189 Baumgarten gilt als Mitbegründerin der psychotechnischen „Charakterologie“, vgl. u.a. Baumgarten 1927a, vgl. Baumgarten 1933. In Kapitel 11 und 12 wird aufgezeigt, wie die Charakterlogie um 1930 zunehmend die Psychotechnik prägte und in der Folge die nationalsozialistische Anwendungspraktik von Glückstechniken u. a. am Beispiel der transnationalen Carl Huter-Rezeption beeinflusste. Vgl. u. a. Wartegg 1939.

190 Vgl. Patzel-Mattern 2010, 62ff. Mehr zur Anwendung der Psychotechnik im Ersten Weltkrieg und zur Etablierung in der Weimarer Republik vgl. u. a. Jaeger und Staeuble 1981. 
Doch die Erfolgsgeschichte im demokratischen System der Weimarer Republik war kurzfristig. Interessenskonflikte, Misstrauensvoten, mangelnde wissenschaftliche Objektivität und Überprüfbarkeit der Auswahlverfahren, betriebsinterner Machtmissbrauch seitens der Psychotechniker*innen beziehungsweise der Unternehmen bis hin zu Partizipationsverweigerungen seitens der Gewerkschaftsvertreter^innen und Arbeiter^innen waren ab Mitte der 1920er Jahre die Folgen. ${ }^{191}$ Mit der Weltwirtschaftskrise 1929, den steigenden Arbeitslosenzahlen und der regierungspolitischen Radikalisierung durch die Wahlsiege der Nationalsozialisten seit 1930 erfuhren der Verwendungszweck der Psychotechnik und das ihr inhärente Glückswissen einen drastischen ideologischen Wandel. ${ }^{192}$

\subsection{Fazit}

Das angewandte Glückswissen in der europaweit rezipierten industriellen Psychotechnik der 1920er Jahre kann exemplarisch für eine Politik der ,Glückskulturen' stehen, die von Brüchen, Kontinuitäten und Ambivalenzen gekennzeichnet ist. So war Eliasberg bei weitem nicht der einzige, der in der neuen Psychotechnik ein utilitaristisches Glückspotential erkannte, um ein demokratisches Zusammenspiel aus sozialpolitischer und staatlicher Verantwortlichkeit, wirtschaftlicher Produktivität und „sozialer Harmonie“ zu schaffen.

Konservativen Sozialromantikern, wie dem deutsch-jüdischen Ökonomen Richard Ehrenbert (1857-1929), aber auch rechten, oftmals völkisch-nationalistischen und antisemitischen Arbeitswissenschaftlern, wie Fritz Giese oder dem Betriebssoziologen Goetz Briefs (1889-1974) ${ }^{193}$, schwebten eine nostalgisch-verklärte Rückkehr in ein vorindustrielles Zeitalter vor, in welchem beispielsweise bei Ehrenbert eine „Werkgemeinschaft“194 eine individualisierte und kapitalistische Wirtschaftlichkeit zugunsten einer wiederzugewinnenden „Arbeitsfreude“ ablösen sollte. ${ }^{195}$

191 Vgl. Patzel-Mattern 2010. Zu den Gründen des Scheiterns vgl. Gründe für das Scheitern der industriellen Psychotechnik. In: Patzel-Mattern 2010, 229-252.

192 S. Kap. 11 und 12.

193 Briefs gründete 1928 das „Institut für Betriebssoziologie und soziale Betriebslehre“ an der Technischen Universität Berlin. 1934 wurde es von den Nationalsozialisten geschlossen, vgl Hinrichs, 1981, 265f. Zu Briefs ,sozialer Betriebspolitik‘ vgl. Rabinbach 1990a, 336f.

194 Zum Begriff der „Werkgemeinschaft“ vgl. u. a. Albrecht 1929, Vorweck und Dunkmann 1928. 195 Vgl. Rabinbach 1990a, 335. 
Vor diesem Hintergrund soll im folgenden Kapitel der Wandel der Arbeitsmoral im Übergang von der Zeit der Weimarer Republik in die NS-Zeit weiterführend untersucht werden. 